\title{
SOME EINSTEIN SPACES WITH CONFORMALLY SEPARABLE FUNDAMENTAL TENSORS
}

\author{
BY \\ YUNG-CHOW WONG(')
}

1. Introduction. When the fundamental tensor $\left({ }^{2}\right)$ of a Riemannian $m$ space $\left(^{(3)} V_{m}\right.$ is of the form ( $\left.{ }^{4}\right)$

$$
*_{\alpha \beta}=\left[\begin{array}{cc}
\rho^{-2} g_{i j} & 0 \\
0 & \sigma^{-2} g_{p q}
\end{array}\right]
$$

$$
\begin{array}{r}
\alpha, \beta, \gamma, \delta, \epsilon=1, \cdots, m, \\
i, j, k, l=1, \cdots, n, \\
p, q, r, s=n+1, \cdots, m,
\end{array}
$$

where

$$
\begin{aligned}
\rho & =\rho\left(x^{\alpha}\right), & \sigma & =\sigma\left(x^{\alpha}\right), \\
g_{i j} & =g_{i j}\left(x^{k}\right), & g_{p q} & =g_{p q}\left(x^{r}\right),
\end{aligned}
$$

it is said to be conformally separable of the type $(n, m-n)$; the tensors ${ }^{*} g_{i j}=\rho^{-2} g_{i j}$ and ${ }^{*} g_{p q}=\sigma^{-2} g_{p q}$, with $x^{r}$ and $x^{k}$, respectively, as parameters, are called its component tensors. We shall say that the tensor (1.1) is properly or improperly conformally separable according as $\partial_{p} \rho \neq 0, \partial_{i} \sigma \neq 0\left({ }^{5}\right)$ are satisfied or not satisfied.

The tensor (1.1) as a generalization of the ordinarily separable tensor $\left[1\right.$, p. 124] $\left(^{(}\right)$was recently introduced by Yano [14], where he proved that in a $V_{m}$ with fundamental tensor

$$
*_{g_{\alpha \beta}}=\left[\begin{array}{cc}
* g_{i j} & 0 \\
0 & *{ } g_{p q}
\end{array}\right]
$$

Presented to the Society, October 31, 1942; received by the editors November 24, 1941.

(1) Most of the results in this paper were obtained while I was a Chinese Ying-Keng Funds Student visiting Princeton University, for the courtesy of whose authorities, especially Professor L. P. Eisenhart, I wish to express my most sincere thanks. I wish also to thank Professor D. J. Struik of Massachusetts Institute of Technology for the conversations we had from time to time during the preparation of the manuscript.

(2) Fundamental tensors are always supposed to be nonsingular, though not necessarily definite. All functions appearing in this paper are assumed to have differentiability properties adequate to the part they play in the discussion.

(3) We denote by $V, S, E$ a Riemannian space, a space of constant curvature, and an Einstein space, respectively. The dimensionality is denoted, if necessary, by an index at the lower right-hand corner.

(4) An index has the same range throughout this paper. An index which appears twice in an expression is to be summed over the appropriate range. A free index of a tensor equation assumes each value of its range. A numerical index at the upper right-hand corner of a letter means an exponential, except in the case of the coordinates $x^{\alpha}, x^{k}$ or $x^{r}$.

(b) We use the notation $\partial_{\alpha} \equiv \partial / \partial x^{\alpha}$.

(8) Such a reference is made to the literatures listed at the end of this paper. 
$n>1$, the subspaces $x^{r}=$ const. are totally umbilical, if and only if $*_{g_{i j}}$ is of the form $\left[\rho\left(x^{\alpha}\right)\right]^{-2} g_{i j}\left(x^{k}\right)$. He also proved that if a conformally separable tensor represents $\left({ }^{7}\right)$ an $S_{m}$ (that is, an $m$-space of constant curvature), then each of its component tensors, if it is of dimension greater than 2, represents $S$ 's.

By definition, an Einstein space $E$ is a $V$ whose Ricci and fundamental tensors differ only by a scalar factor $\left.{ }^{8}\right)$. The result mentioned at the end of the last paragraph no longer holds if $S$ is replaced by $E$, although an $S$ is a special $E$. In this paper we present a complete study of the conformally separable tensor which represents an $E_{m}$ and each of whose component tensors either is of dimension less than 3 or represents $E$ 's. It is found that the construction of such a conformally separable tensor is invariably reduced to that of the fundamental tensor $g_{i j}$ of an $E_{n}$ or a $V_{2}$ for which the following equation admits a solution $\left({ }^{9}\right)$ for $y$ :

$$
y_{, i j}=-I g_{i j}
$$

where the comma denotes covariant differentiation with respect to $g_{i j}$, and $I$ is an unspecified scalar. We shall be content with this result, because the latter problem has already been considered in detail by Brinkmann $[2,3]$ in his study of $E$ 's which are conformal to each other.

In $\$ 2$, some results concerning the differential equation (1.3) are given. In $\S 3$, we find the expressions for the Riemann and Ricci tensors of the tensor (1.1) in terms of those for the same-named tensors of its component tensors. Concerning a properly conformally separable tensor of the type $(n>1$, $m-n=1$ ), which we consider in $\$ 4$, we prove (1) that if an $E_{m}$ admits a oneparameter family of totally umbilical hypersurfaces, then they are conformal to one another and each of them has constant scalar curvature (Theorem 4.1); and (2) that a one-parameter family of conformal $E_{n}$ 's with fundamental tensors $\left[\rho\left(x^{k}, x^{m}\right)\right]^{-2} g_{i j}\left(x^{k}\right)$ can in general be imbedded isometrically in an $E_{n+1}$ as totally umbilical hypersurfaces (Theorem 4.2). $\$ \$ 5$ and 6 are devoted to the study of a properly conformally separable tensor ${ }^{*} g_{\alpha \beta}$ of the type $(n>1, m-n>1)$ which represents an $E_{m}$ and each of whose component tensors is either of dimension 2 or represents $E$ 's. By means of Theorem 5.1 on a certain system of differential equations, we show that ${ }^{*} g_{\alpha \beta}$ is conformal to an ordinarily separable tensor of the type $(n, m-n)$ (Theorem 5.2). This result enables us to prove that the component tensors of ${ }^{*} g_{\alpha \beta}$ have the property that, either each of them represents $E$ 's or $S_{2}$ 's, or $n=m-n=2$ and neither of them represents $S_{2}$ 's (Theorem 6.2). Characteristic properties of ${ }^{*} g_{\alpha \beta}$ are then derived (Theorems 6.3 and 6.4 ), showing how the construction of ${ }^{*} g_{\alpha \beta}$ depends on that of the

(7) We sometimes find it convenient to express the fact that $g_{i j}$ is the fundamental tensor of an $S$ (or $E$ ) by saying that $g_{i j}$ represents an $S$ (or $E$ ).

(8) A $V_{2}$ is always an $E_{2}$, and an $E_{3}$ is identical with an $S_{3}$ [13]. For convenience, we agree that whenever we speak of an $E$, it is understood that $E$ is of dimension greater than 2 .

${ }^{(9)} \mathrm{By}$ "solution" we always mean non-constant solution. 
fundamental tensor of an $E_{n}$ or a $V_{2}$ which admits a solution of (1.3).

The discussion of improperly conformally separable tensors is much easier and is carried out in $\$ \$ 7$ and 8 . In $\$ 9$, the theorem of Yano concerning an $S_{m}$ with conformally separable fundamental tensor is extended, and the paper ends at $\$ 10$ with some canonical forms for the conformally separable tensors of the type $(2,2)$ which represents $E_{4}$ 's.

We conclude this introduction with the following remarks. Since the component tensor ${ }^{*} g_{i j}$ of the tensor (1.1) can be written as

$$
{ }^{*} g_{i j}=\left[\frac{\rho\left(x^{k}, x^{r}\right)}{\rho\left(x^{k}, x_{0}^{r}\right)}\right]^{-2}\left[\rho\left(x^{k}, x_{0}^{r}\right)\right]^{-2} g_{i j}
$$

where $x_{0}^{r}$ are certain fixed values of $x^{r}$, there is no loss of generality in assuming that the function $\rho$ is such that

$$
\rho\left(x^{k}, x_{0}^{r}\right)=1 \text {; }
$$

in particular, if $\partial_{p} \rho=0$, we may assume that $\rho=1$. This assumption will be made whenever it is desirable. A similar remark holds for the function $\sigma$. Finally, the fundamental tensor of every $V_{2}$ referred to orthogonal coordinate curves is conformally separable, and for this reason we shall always suppose that $m>2$.

\section{Preliminaries}

2. The equation $y_{, i j}=-I g_{i j}$. In what follows we have frequent occasions to meet the following differential equation in the unknown scalar $y$ :

$$
y_{, i j}=-I g_{i j}, \quad f, h, i, j, k, l=1, \cdots, n(>1),
$$

where $I$ is an unspecified scalar and the comma denotes covariant differentiation with respect to the fundamental tensor $g_{i j}$. This equation has been considered by several authors for different purposes (Brinkmann [3, pp. 121124]; Fialkow [7, pp. 426-427; 8, pp. 471-473]; Yano [16]; Delgleize [4]). Here we confine ourselves to the case when the Ricci tensor $R_{i j}$ of the $V_{n}$ with fundamental tensor $g_{i j}$ satisfies

$$
R_{i j}=-(n-1) a g_{i j},
$$

$$
a=\text { a scalar, }
$$

that is, when $V_{n}$ is a $V_{2}(a)$ or an $E_{n}(a)\left({ }^{10}\right)$. In the latter case, (2.2) implies that $a=$ const. $[5$, p. 93, Exercise 5$]$.

We first find a geometric meaning of (2.1). The Ricci tensors $R_{i j}$ and $\bar{R}_{i j}$ of $g_{i j}$ and $\bar{g}_{i j}=y^{-2} g_{i j}$ are connected by $[5$, p. 90 , (28.6)]

$$
\bar{R}_{i j}=R_{i j}-(n-2) \frac{y_{, i j}}{y}+g_{i j}\left[-\frac{g^{h k} y_{, h k}}{y}+(n-1) \frac{g^{h k} y_{, h} y_{, k}}{y^{2}}\right] .
$$

(10) We denote an $E$ or $S$ of scalar curvature $a$ by $E(a)$ or $S(a)$, respectively. 
From this it follows at once that

THEOREM 2.1. Given an $E_{n}(n>2)$ with fundamental tensor $g_{i j}$, the $V_{n}$ with fundamental tensor $y^{-2} g_{i j}$ is also an $E_{n}$, if and only if $y$ satisfies (2.1).

By definition, a $V_{n}$ is an $S_{n}$ if the Riemann tensor of $V_{n}$ is of the form

$$
R_{i j k}^{l}=a\left(\delta_{j}^{l} g_{i k}-\delta_{k i j}^{l} g_{i j}\right),
$$$$
a=\text { const. }
$$

A consequence of this is that an $S_{n}$ is necessarily an $E_{n}$. The Riemann tensors $R_{i j k}^{l}$ and $\bar{R}_{k j \mathrm{k}}^{l}$ of $g_{i j}$ and $\bar{g}_{i j}=y^{-2} g_{i j}$ are connected by [5, p. 90, (28.10)]

$$
\begin{aligned}
\bar{R}_{i j k}^{l}=R_{i j k}^{l}+ & \left(\delta_{j}^{l} y_{, i k}-\delta_{k}^{l} y_{, i j}\right) y^{-1}+g^{h l}\left(y_{, h j} g_{i k}-y_{, h k} g_{i j}\right) y^{-1} \\
& -g^{f h} y_{, f} y_{, h}\left(\delta_{j}^{l} g_{i k}-\delta_{k}^{l} g_{i j}\right) y^{-2} .
\end{aligned}
$$

From (2.4), (2.5) and Theorem 2.1 it can easily be proved that

THEOREM 2.2. Theorem 2.1 remains true when $E_{n}$ is replaced by $S_{n}$.

We now suppose that (2.1) has a solution $y$. The integrability condition of (2.1) is

$$
R_{i j k}^{l} y_{, l}=y_{, i j k}-y_{, i k j}=-g_{i j} I_{, k}+g_{i k} I_{, j} \text {. }
$$

Transvecting this by the contravariant components $g^{i j}$ of $g_{i j}$, we have

$$
R_{k}^{l} y_{, l}=-(n-1) I_{, k},
$$

where $R_{k}^{l}=g^{h l} R_{h k}$. When (2.2) is satisfied, (2.7) becomes

$$
a y_{, k}=I_{, k},
$$

from which it follows that

$$
I=I(y), \quad a=a(y)=d I / d y .
$$

By differentiating $g^{i j} y_{, i} y_{, j}$ and then making use of (2.1) and (2.9), we obtain so that

$$
\left(g^{i j} y_{, i} y_{, j}\right)_{, k}=-2 I(y) y_{, k},
$$

$$
g^{i j} y_{, i} y_{, j}=-2 \int I(y) d y \equiv-2 J(y) .
$$

If $V_{n}$ is an $E_{n}$ or $S_{2}, a=$ const. For this case, it follows from (2.9) and (2.10) that

$$
I(y)=a y+f, \quad g^{i j} y_{, i} y_{, j}=-\left(a y^{2}+2 f y+\bar{a}\right),
$$

where $f$ and $\bar{a}$ are two constants. Summing up the preceding results we have TheOREM 2.3. Let $a V_{n}$ admit a solution $y$ of (2.1). Then equations (2.9) and 
(2.10) hold if $V_{n}$ is an $V_{2}$; and equations (2.9), (2.10) and (2.11) hold if $V_{n}$ is an $E_{n}(n>2)$ or $S_{2}$.

To find a meaning of the constant $\bar{a}$ appearing in (2.11), we use (2.1), (2.2) and $(2.11)_{2}$ in (2.3), and obtain

$$
\bar{R}_{i j}=-(n-1) \bar{a} y^{-2} g_{i j} .
$$

This shows that $\bar{g}_{i j}=y^{-2} g_{i j}$ is the fundamental tensor of an $E_{n}(\bar{a})$ or $S_{2}(\bar{a})$. Hence we have

THEOREM 2.4. If $g_{i j}$ is the fundamental tensor of an $E_{n}(a)$ or $S_{2}(a)$ and $y$ is a solution of (2.1), then $y^{-2} g_{i j}$ is the fundamental tensor of an $E_{n}(\bar{a})$ or $S_{2}(\bar{a})$, where $\bar{a}$ is determined from $(2.11)_{2}$.

We can also prove that

THEOREM 2.5. If $g_{i j}=y^{2} \bar{g}_{i j}$ is the fundamental tensor of an $S_{2}$ and $y$ satisfies the equation

$$
\left(\frac{1}{y}\right)_{l i j}=-\bar{I} \bar{g}_{i j}
$$

where the solidus denotes covariant differentiation with respect to $\bar{g}_{i j}$ and $\bar{I}$ is an unspecified scalar, then $\bar{g}_{i j}$ is also the fundamental tensor of an $S_{2}$.

Proof. On account of the preceding theorem, we need show only that (2.1) is satisfied. Now it can be easily verified that if $w_{i}$ is any vector, then its covariant derivatives $w_{i / j}$ and $w_{i, j}$ taken with respect to $\bar{g}_{i j}$ and $g_{i j}=y^{2} \bar{g}_{i j}$, respectively, are related by

$$
w_{i / j}=w_{i, j}+\left(w_{, i} y_{, j}+w_{, j} y_{, i}\right) y^{-1}-\bar{g}_{i j} \bar{g}^{h k} y_{, h} y_{, k} .
$$

Therefore we have, from hypothesis and by (2.13),

$$
\begin{aligned}
-\bar{I} \bar{g}_{i j} & =\left(\frac{1}{y}\right)_{l i j}=-\frac{y_{/ i j}}{y^{2}}+\frac{2 y_{/ i} y_{/ j}}{y^{3}} \\
& =-\frac{1}{y^{2}}\left(y_{, i j}+\frac{2 y_{, i} y_{, j}}{y}-\bar{g}_{i j} \bar{g}^{h k} y_{, h} y_{, k}\right)+\frac{2 y_{, i} y_{, i}}{y^{3}} \\
& =-\frac{y_{, i j}}{y^{2}}+\bar{g}_{i j} \frac{\bar{g}^{h k} y_{, h} y_{, k}}{y^{2}}
\end{aligned}
$$

which shows that $y$ satisfies an equation of the form (2.1), as was to be proved.

Canonical forms for the fundamental tensor of an $E_{n}$ which admits a solution $y$ of equation (2.1) have been given by Brinkmann [3]. We shall not enter into the detail of his results, but merely mention the main fact that the construction of such a canonical form depends, according as $g^{i j} y_{, i} y_{, j} \neq 0$ or $=0$, on the fundamental tensor of an arbitrary $E_{n-1}$ or on the fundamental 
tensor of an $E_{n-2}$ which contains a parameter and satisfies certain differential equations.

If a $V_{n}$ with nonzero scalar curvature $a$, which may or may not be constant, admits a solution $y$ of (2.1), then it follows from (2.9) and (2.10) that $g^{i j} y_{, i} y_{, j} \neq 0$. Consequently, we can show, by following Brinkmann's method, that the fundamental form of $V_{2}$ can be reduced to

$$
-\frac{\left(d x^{1}\right)^{2}}{2 J\left(x^{1}\right)}+2 e J\left(x^{1}\right)\left(d x^{2}\right)^{2}
$$

where $e= \pm 1$ and $J\left(x^{1}\right)$ is defined by (2.10). Conversely, if the fundamental form of a $\bar{V}_{2}$ is of the form (2.14), where $J\left(x^{1}\right)$ is any function of $x^{1}$, then $y=x^{1}$ is a solution of (2.1).

Finally, we remark that for any $V_{n}$ the following particular case of equation (2.1):

$$
y_{, i j}=0
$$

admits a solution, if and only if $V_{n}$ has a family of parallel totally geodesic hypersurfaces, or what amounts to the same thing, a field of parallel vectors.

3. Fundamental relations. From (1.1), it is evident that the components of the conformally separable tensor ${ }^{*} g_{\alpha \beta}$ and those of its component tensors ${ }^{*} g_{i j}$ and ${ }^{*} g_{p q}$ are connected by

$$
\begin{aligned}
& { }^{*} g_{i p}=*^{i p}=0, \quad *_{g}^{i j} *_{g_{i k}}=\delta_{k}^{j}, \quad *^{p q} *^{p} g_{p r}=\delta_{r}^{q}, \\
& * g_{i j}=\rho^{-2} g_{i j}, \quad * g^{i j}=\rho^{2} g^{i j}, \quad g^{i j} g_{i k}=\delta_{k}^{i}, \\
& * g_{p q}=\sigma^{-2} g_{p q}, \quad \quad *^{p q}=\sigma^{2} g^{p q}, \quad g^{p q} g_{p r}=\delta_{r}^{q},
\end{aligned}
$$

where $\delta_{k}^{j}$ and $\delta_{r}^{q}$ are Kronecker deltas. Denoting by ${ }^{*} \Gamma_{\alpha \beta}^{\gamma},{ }^{\prime} \Gamma_{y,}^{k},{ }^{\prime} \Gamma_{p q}^{r}$ the Christoffel symbols of the second kind for the fundamental tensors ${ }^{*} g_{\alpha \beta},{ }^{*} g_{i j}$, ${ }^{*} g_{p q}$, respectively, we have $[14,(3.3)]$

$$
\begin{array}{lll}
* \Gamma_{i j}^{k}={ }^{\prime} \Gamma_{i j,}^{k}, & * \Gamma_{i j}^{p}={ }^{*} g_{i j \rho} \rho^{p}, & * \Gamma_{p i}^{k}=-\rho_{p} \delta_{j i}^{k}, \\
{ }^{*} \Gamma_{p q}^{r}={ }^{\prime} \Gamma_{p q}^{r}, & { }^{*} \Gamma_{p q}^{k}={ }^{*} g_{p q} \sigma^{k}, & * \Gamma_{i q}^{r}=-\sigma_{i} \delta_{q}^{r},
\end{array}
$$

where

$$
\begin{array}{ll}
\rho_{p}=\partial_{p} \log \rho, & \sigma_{i}=\partial_{i} \log \sigma, \\
\rho^{p}={ }^{*} g^{p q} \rho_{q}, & \sigma^{i}={ }^{*} g^{i j} \sigma_{j} .
\end{array}
$$

The Riemann tensor of ${ }^{*} g_{\alpha \beta}$ is defined by

$$
{ }^{*} R_{\alpha \beta \gamma}^{e}=\partial_{\beta} * \Gamma_{\alpha \gamma}^{e}-\partial_{\gamma}{ }^{*} \Gamma_{\alpha \beta}^{\epsilon}+{ }^{*} \Gamma_{\alpha \gamma}^{\delta} * \Gamma_{\delta \beta}^{e}-{ }^{*} \Gamma_{\alpha \beta}^{\delta}{ }^{*} \Gamma_{\delta \gamma}^{\epsilon}
$$


If the values of ${ }^{*} \Gamma_{\alpha \beta}^{\gamma}$ given by (3.2) are used, then with some calculation we find

$$
\begin{aligned}
& * R_{i j k}^{l}={ }^{\prime} R_{i j k}^{l}-\rho^{p} \rho_{p}\left(\delta_{j}^{l} *_{g_{i k}}-\delta_{k}^{l}{ }^{*} g_{i j}\right), \\
& { }^{*} R_{i j p}^{l}=\left(\delta_{j}^{l} \delta_{i}^{k}-{ }^{*} g{ }^{l k}{ }^{*} g_{i j}\right)\left(\partial_{k} \rho_{p}+\rho_{p} \sigma_{k}\right)=-*^{*} R_{i p j}^{l}, \\
& { }^{*} R_{p j k}^{l}=\delta_{j}^{l}\left(\partial_{k} \rho_{p}+\rho_{p} \sigma_{k}\right)-\delta_{k}^{l}\left(\partial_{j} \rho_{p}+\rho_{p} \sigma_{j}\right), \\
& { }^{*} R_{i p q}^{l}=0 \\
& { }^{*} R_{p q k}^{l}=\delta_{k \rho}^{l}\left(\frac{1}{\rho}\right)_{; p q}+{ }^{*} g_{p q}{ }^{*} g^{l j} \sigma\left(\frac{1}{\sigma}\right)_{; k j}=-{ }^{*} R_{p k q}^{l}, \\
& * R_{p q r}^{l}={ }^{*} g^{h l}\left[{ }^{*} g_{r p}\left(\partial_{q} \sigma_{h}+\rho_{q} \sigma_{h}\right)-{ }^{*} g_{p q}\left(\partial_{r} \sigma_{h}+\rho_{r} \sigma_{h}\right)\right],
\end{aligned}
$$

where, as well as in what follows, the semi-colon denotes covariant differentiation with respect to ${ }^{*} g_{i j}$ or ${ }^{*} g_{p q}$, and ' $R_{i j \mathrm{k}}^{l}$ is the Riemann tensor of ${ }^{*} g_{i j}$. In deriving (3.4) use has been made of the following formulae:

$$
\begin{gathered}
\Gamma_{i j}^{l}=\Gamma_{i j}^{l}-\delta_{i \rho_{j}}^{l}-\delta_{j \rho_{i}}^{l}+g_{i j} g^{l k} \rho_{k} \quad[5, \text { p. } 89(28.3)], \\
\partial_{k}\left({ }^{*} g_{p q} \sigma^{l}\right)={ }^{*} g_{p q}\left(-2 \sigma_{k} \sigma^{l}-{ }^{*} g^{l i}{ }^{l i} \Gamma_{k i}^{j} \sigma_{j}-{ }^{\prime} \Gamma_{k i}^{l}{ }^{i}+{ }^{*}{ }^{l j} \partial_{k} \sigma_{j}\right), \\
\partial_{q} \rho_{p}-{ }^{\prime} \Gamma_{p q}^{r} \rho_{r}-\rho_{p} \rho_{q}=-\rho\left(\frac{1}{\rho}\right)_{; p q},
\end{gathered}
$$

where $\Gamma_{i j}^{l}$ denotes the Christoffel symbols of the second kind for $g_{i j}$. The expressions for the remaining components of $* R_{\alpha \beta \gamma}^{e}$ are obtained from (3.4) by interchanging the two sets of indices $(i, j, k, l)$ and $(p, q, r, s)$. We remark that (3.4) can be shown to be identical with the Gauss-Codazzi-Ricci equations [5, pp. 162-163, (47.11), (47.12), (47.14)] for the subspaces $x^{r}=$ const. in the $V_{m}$ with fundamental tensor ${ }^{*} g_{\alpha \beta}$.

The components of the Ricci tensor ${ }^{*} R_{\alpha \beta}={ }^{*} R_{\alpha \beta \gamma}^{\gamma}$ of ${ }^{*} g_{\alpha \beta}$ are readily found from (3.4) by contraction; they are

$$
\begin{aligned}
-{ }^{*} R_{i p}= & (n-1) \partial_{i} \rho_{p}+(m-n-1) \partial_{p} \sigma_{i}+(m-2) \rho_{p} \sigma_{i}, \\
{ }^{*} R_{i j}= & ' R_{i j}+(m-n) \sigma\left(\frac{1}{\sigma}\right)_{i i j} \\
& +g_{i j} g^{p q}\left[\rho\left(\frac{1}{\rho}\right)_{; p q}+(n-1) \rho^{2}\left(\frac{1}{\rho}\right)_{; p}\left(\frac{1}{\rho}\right)_{; q}\right], \\
{ }^{*} R_{p q}= & ' R_{p q}+n \rho\left(\frac{1}{\rho}\right)_{; p q} \\
& +g_{p q} g^{i j}\left[\sigma\left(\frac{1}{\sigma}\right)_{; i j}+(m-n-1) \sigma^{2}\left(\frac{1}{\sigma}\right)_{; i}\left(\frac{1}{\sigma}\right)_{; j}\right],
\end{aligned}
$$

where ' $R_{i j} \quad\left({ }^{\prime} R_{p q}\right)$ is zero or is the Ricci tensor of ${ }^{*} g_{i j}\left({ }^{*} g_{p q}\right)$ according as ${ }^{*} g_{i j}\left({ }^{*} g_{p q}\right)$ is of dimension 1 or greater than 1 . 
II. CONFORMALly SEPARABLE TENSOR OF THE TYPE $(n>1, m-n=1)\left({ }^{11}\right)$

4.1. Scalar curvatures of totally umbilical hypersurfaces in an $E_{m}$. A conformally separable tensor of the type $(n>1, m-n=1)$ may be taken as

$$
*_{g_{\alpha \beta}}=\left[\begin{array}{cr}
\rho^{-2} g_{i j} & 0 \\
0 & e \sigma^{-2}
\end{array}\right], e= \pm 1, \quad \alpha, \beta, \gamma, \delta=1, \cdots, m(=n+1),
$$

for, we may suppose that $\pm g_{m m}$ has been absorbed in $\sigma^{-2}$. From (3.6) it follows that the condition

$$
{ }^{*} R_{\alpha \beta}=-(m-1) c^{*} g_{\alpha \beta}, \quad c=\text { const., }
$$

for the tensor (4.1) to represent an $E_{m}(c)$ is

$$
\begin{gathered}
\partial_{i} \rho_{m}+\rho_{m} \sigma_{i}=0, \\
{ }^{\prime} R_{i j}+\sigma\left(\frac{1}{\sigma}\right)_{; i j}+{ }^{*} g_{i j}{ }^{*} g^{m m}\left[\rho\left(\frac{1}{\rho}\right)_{; m m}+(m-2) \rho^{2}\left(\frac{1}{\rho}\right)_{; m}\left(\frac{1}{\rho}\right)_{; m}\right] \\
=-(m-1) \\
(m-1) \rho\left(\frac{1}{\rho}\right)_{; m m}+{ }^{*} g_{m m}{ }^{*} g^{i j} \sigma\left(\frac{1}{\sigma}\right)_{; i j}=-(m-1) c^{*} g_{m m},
\end{gathered}
$$

where, we repeat, ${ }^{*} g^{m m}=1 /{ }^{*} g_{m m}=e \sigma^{2},{ }^{\prime} R_{i j}$ is the Ricci tensor of ${ }^{*} g_{i j}$, and the semi-colon denotes covariant differentiation with respect to ${ }^{*} g_{i j}$ or ${ }^{*} g_{m m}$.

If we write

$$
{ }^{*} g^{i j} R_{i j}=-(m-1)(m-2) * a\left(x^{k}, x^{m}\right),
$$

then, by definition, ${ }^{*} a\left(x^{k}, x^{m}\right)$ is the scalar curvature of ${ }^{*} g_{i j}=\rho^{-2} g_{i j}$. We shall now prove that ${ }^{*} a\left(x^{k}, x^{m}\right)$ is independent of $x^{k}$.

Transvecting $(4.3)_{2}$ and $(4.3)_{3}$ by ${ }^{*} g^{i j}$ and ${ }^{*} g^{m m}$ respectively, and taking account of (4.4), we obtain

$$
\begin{aligned}
& -(m-1)(m-2) * a\left(x^{k}, x^{m}\right)+{ }^{*} g^{i j} \sigma\left(\frac{1}{\sigma}\right)_{i i j} \\
& +(m-1)^{*} g^{m m}\left[\rho\left(\frac{1}{\rho}\right)_{; m m}+(m-2) \rho^{2}\left(\frac{1}{\rho}\right)_{; m}\left(\frac{1}{\rho}\right)_{; m}\right] \\
& \quad=-(m-1)^{2} c \\
& \quad(m-1)^{*} g^{m m} \rho\left(\frac{1}{\rho}\right)_{; m m}+{ }^{*} g^{i j} \sigma\left(\frac{1}{\sigma}\right)_{i i j}=-(m-1) c .
\end{aligned}
$$

When the latter equation is subtracted from the former, and $e \sigma^{2}$ is used in place of ${ }^{*} g^{m m}$, the result is

(11) In $\$ \$ 4.1$ and 4.2 we do not confine ourselves to properly conformally separable tensors, but a complete discussion of improperly conformally separable tensors is reserved for $\$ \S 7$ and 8. 


$$
* a\left(x^{k}, x^{m}\right)-e\left(\sigma \rho_{m}\right)^{2}=c .
$$

If $\partial_{m} \rho=0$, this reduces to

$$
* a\left(x^{k}, x^{m}\right)=c .
$$

If $\partial_{m} \rho \neq 0$, then equation $(4.3)_{1}$ can be written

$$
\frac{\partial_{i} \rho_{m}}{\rho_{m}}+\sigma_{i}=0, \text { that is, } \partial_{i} \log \left(\sigma \rho_{m}\right)=0,
$$

which gives

$$
\sigma \rho_{m}=z\left(x^{m}\right),
$$

where $z$ is a function of $x^{m}$ alone. Therefore equation (4.6) becomes

$$
* a\left(x^{k}, x^{m}\right)-e\left[z\left(x^{m}\right)\right]^{2}=c .
$$

This equation and $\left(4.6^{\prime}\right)$ show that ${ }^{*} a\left(x^{h}, x^{m}\right)$ does not depend on $x^{k}$, as was to be proved. Hence

THEOREM 4.1. If a conformally separable tensor of the type $(n>1, m-n=1)$ represents an $E_{m}$, then its first component tensor represents $V_{n}$ 's of constant scalar curvatures.

And geometrically $\left({ }^{12}\right)$,

THEOREM 4.1'. If in an $E_{m}$ there exists a one-parameter family of totally umbilical hypersurfaces, then these hypersurfaces are conformal to one another and each of them has constant scalar curvature. If, in particular, the family consists of totally geodesic hypersurfaces, then they are isometric to one another and their constant scalar curvatures are all equal to the scalar curvature of $E_{m}$.

The latter part of this theorem follows from (4.6').

4.2. An imbedding theorem. Continuing our discussion, we now write ${ }^{*} a\left(x^{m}\right)=* a\left(x^{k}, x^{m}\right)$, and proceed to prove the following

THEOREM 4.2. In order that the conformally separable tensor (4.1) with $\partial_{m} \rho \neq 0$ may represent an $E_{m}(c)$ and its first component tensor ${ }^{*} g_{i j}=\rho^{-2} g_{i j} E_{n}$ 's or $S_{2}$ 's of scalar curvatures * $a\left(x^{m}\right)$, it is necessary and sufficient that when

$$
\rho\left(x^{k}, x_{0}^{m}\right)=1
$$

is assumed, the equations

(12) A more general result can be obtained by using the Gauss-Codazzi equations of a $V_{n}$ in an $E_{n+1}$. Indeed we can prove that if an $E_{n+1}$ has a totally umbilical hypersurface $V_{n}$, then the scalar curvature of $V_{n}$ is constant. But we shall not go farther with this result, because Theorem 4.1' serves only as a preliminary to the imbedding Theorem 4.2'. 


$$
\begin{aligned}
R_{i j} & =-(n-1) a g_{i j}, \\
\rho_{, i j} & \sim g_{i j}, \\
e \sigma^{-2} & =\frac{\left(\rho_{m}\right)^{2}}{{ }^{*} a\left(x^{m}\right)-c}
\end{aligned}
$$$$
a=\text { const. }
$$

be satisfied.

Here we write (4.11) to mean the equation $\rho_{, i j}=-\omega g_{i j}$, where $\omega$ is an unspecified scalar. This notation will be used whenever desirable; it enables us to avoid the unnecessary introduction of many symbols to represent scalar factors of proportionality.

We know that in a $V_{m}$ the hypersurfaces $x^{m}=$ const., whose (first) fundamental tensors are nonsingular, are totally umbilical if and only if the fundamental tensor of $V_{m}$ can be reduced to the form $(4.1)$ [5, pp. 144, 182]. We also know from Theorem 2.1 that when $n>2$ and (4.9), is satisfied, equations (4.10), (4.11) are the conditions that

$$
{ }^{\prime} R_{i j}=-(n-1)^{*} a\left(x^{m}\right)^{*} g_{i j},
$$

that is that ${ }^{*} g_{i j}=\rho^{-2} g_{i j}$ represents $E_{n}$ 's. Accordingly Theorem 4.2 may be stated geometrically as follows.

THEOREM 4.2'. Let $c$ be any constant and let ${ }^{*} g_{i j}=\left[\rho\left(x^{k}, x^{m}\right)\right]^{-2} g_{i j}\left(x^{k}\right)$ with $x^{m}$ as parameter and $\partial_{m} \rho \neq 0$ represent $\infty^{1} V_{n}$ 's whose scalar curvatures are not all equal to $c$. If the $V_{n}$ 's are $E_{n}$ 's $(n>2)$, there exists an $E_{n+1}(c)$ in which they are imbedded isometrically as totally umbilical hypersurfaces. If the $V_{n}$ 's are $S_{2}$ 's, a necessary and sufficient condition that they may be imbedded isometrically in an $S_{3}(c)$ as totally umbilical surfaces is that, when $\rho\left(x^{k}, x_{0}^{m}\right)=1$ is assumed, the tensor $\rho_{, i j}$ differ from $g_{i j}$ by a scalar factor. The fundamental tensor of the imbedding $E_{n+1}(c)$ or $S_{3}(c)$, if it exists, is

$$
{ }^{*} g_{\alpha \beta}=\left[\begin{array}{cc}
\rho^{-2} g_{i j} & 0 \\
0 & \left(\partial_{n} \log \rho\right)^{2} /\left[{ }^{*} a\left(x^{m}\right)-c\right]
\end{array}\right],
$$

where ${ }^{*} a\left(x^{m}\right)$ denotes the scalar curvatures of the given $E_{n}$ 's or $S_{2}$ 's.

We shall now prove Theorem 4.2 . Since by hypothesis $\partial_{m} \neq \neq 0$, equations (4.6), (4.7) and (4.8) are consequences of (4.3), as we have seen in \$4.1. If we solve (4.6) for $e \sigma^{-2}$, the result is (4.12). Hence Theorem 4.2 will be proved if we can show that in consequence of $\left({ }^{(1)}\right)(4.7),(4.8),(4.9)$ and (4.13), equations $(4.3)$ reduce to $(4.11)$.

Now equation $(4.3)_{1}$ is satisfied because of (4.7). When (4.13) is used, equation $(4.3)_{2}$ becomes

(13) We observe that equations (4.6)-(4.8) and (4.12) are unaffected by the supposition (4.9). 


$$
\begin{aligned}
\sigma\left(\frac{1}{\sigma}\right)_{i i j}=\{- & (m-1) c+(n-1)^{*} a\left(x^{m}\right) \\
& \left.\quad{ }^{*} g^{m m}\left[\rho\left(\frac{1}{\rho}\right)_{i m m}+(m-2) \rho^{2}\left(\frac{1}{\rho}\right)_{i m}\left(\frac{1}{\rho}\right)_{i m}\right]\right\}^{*} g_{i j},
\end{aligned}
$$

which is evidently equivalent to $(4.5)_{1}$ and

$$
\left(\frac{1}{\sigma}\right)_{i i j} \sim{ }^{*} g_{i j}
$$

But equation $(4.5)_{1}$ is a consequence of $(4.7),(4.8)$ and $(4.3)_{3}$, as is evident from the way in which (4.7) and (4.8) were derived. Thus, because of (4.7), (4.8) and (4.13), equations (4.3) are equivalent to (4.14) and (4.3) 8 . In what follows we shall reduce the latter two equations successively to $(4.16)$, then to (4.20), and finally to (4.11).

Comparing (4.14) and (4.13) with (2.1) and (2.2), it follows from (2.11) that equation (4.14) can be written

$$
\left(\frac{1}{\sigma}\right)_{i i j}=-\left[*^{*} a\left(x^{m}\right) \frac{1}{\sigma}+w\left(x^{m}\right)\right] * g_{i j}
$$

where $w\left(x^{m}\right)$ is a function of $x^{m}$ alone. In virtue of this, equation (4.3), becomes

$$
e \sigma^{2} p\left(\frac{1}{\rho}\right)_{; m m}+c-{ }^{*} a\left(x^{m}\right)-\sigma w\left(x^{m}\right)=0 .
$$

Now

$$
\begin{aligned}
\left(\frac{1}{\rho}\right)_{; m} & =-\frac{\rho_{m}}{\rho} \\
\left(\frac{1}{\rho}\right)_{; m m} & =\partial_{m}\left(-\frac{\rho_{m}}{\rho}\right)-{ }^{\prime} \Gamma_{m m}^{m}\left(-\frac{\rho_{m}}{\rho}\right) \\
& =\frac{\rho_{m}}{\rho}\left(-\partial_{m} \log \frac{\rho_{m}}{\rho}+(1 / 2) \partial_{m} \log { }^{*} g_{m m}\right) \\
& =\frac{\rho_{m}}{\rho} \partial_{m} \log \frac{\rho}{\sigma \rho_{m}}=\frac{\rho_{m}}{\rho}\left[\rho_{m}-\frac{z^{\prime}\left(x^{m}\right)}{z\left(x^{m}\right)}\right]
\end{aligned}
$$

by (4.7), where and in what follows, the prime denotes differentiation. Substituting the above expression in (4.15) and then using (4.7), (4.8) and the equation obtained by differentiating (4.8), we find

$$
w\left(x^{m}\right)=-e z^{\prime}\left(x^{m}\right)=-\frac{{ }^{*} a^{\prime}\left(x^{m}\right)}{2 z\left(x^{m}\right)}=-\frac{{ }^{*} a^{\prime}\left(x^{m}\right)}{2 \sigma \rho_{m}} .
$$


Because of this, equation $\left(4.14^{\prime}\right)$ becomes

$$
\left(\frac{1}{\sigma}\right)_{i i j}=-\frac{1}{\sigma}\left[* a\left(x^{m}\right)-\frac{{ }^{*} a^{\prime}\left(x^{m}\right)}{2 \rho_{m}}\right] * g_{i j} .
$$

This is an equation which, because of (4.7) and (4.8), is equivalent to (4.14) and $(4.3)_{3}$.

We now express (4.16) directly in terms of $\rho$ and $g_{i j}$. To do this we make use of (4.7) and the following formula, which can easily be proved by means of $(3.5)_{1}$ :

$$
\left(\frac{1}{\sigma}\right)_{i i j}=\frac{1}{\rho}\left(\frac{\rho}{\sigma}\right)_{, i j}-\frac{\rho_{, i j}}{\sigma}-g^{h k}\left(\frac{1}{\sigma}\right)_{, h} \rho_{. k} g_{i j},
$$

where as usual the comma denotes covariant differentiation with respect to $g_{i j}$. Equation (4.16) then becomes

$$
\begin{aligned}
{\left[-{ }^{*} a\left(x^{m}\right) \rho_{m}+(1 / 2) * a^{\prime}\left(x^{m}\right)+\right.} & \left.\rho g^{h k}\left(\rho_{m}\right)_{, h} \rho_{, k}\right] g_{i i} \\
& =\rho\left(\partial_{m} \rho\right)_{, i j}-\left(\partial_{m} \rho\right) \rho_{, i j}=\rho^{2} \partial_{m}\left(\frac{\rho_{, i j}}{\rho}\right),
\end{aligned}
$$

because $\left(\partial_{m} \rho\right)_{, i j}=\partial_{m}(\rho, i j)$. On account of (4.9), the scalar curvatures $a={ }^{*} a\left(x_{0}^{m}\right)$ and ${ }^{*} a\left(x^{m}\right)$ of $g_{i j}$ and $\rho^{-2} g_{i j}$ are connected by [5, p. 90, (28.7)]

$$
{ }^{*} a\left(x^{m}\right)=a \rho^{2}+\frac{2}{n} \rho g^{h k} \rho, h k-g^{h k} \rho, h \rho, k \text {. }
$$

By use of this equation and its partial derivative with respect to $x^{m}$, we can easily verify that the coefficient of $g_{i j}$ in (4.18) is

$$
\frac{1}{n} g^{h k}\left[\rho\left(\partial_{m} \rho\right)_{, h k}-\left(\partial_{m} \rho\right) \rho_{, h k}\right] .
$$

This shows that, when (4.9) is supposed, equation (4.18), and hence also (4.16), are equivalent to

$$
\partial_{m}\left(\frac{\rho_{, i j}}{\rho}\right) \sim g_{i j}
$$

Finally, to reduce this to (4.11), we integrate it with respect to $x^{m}$ and obtain

$$
\frac{\rho_{i j}}{\rho}+T_{i j} \sim g_{i j}
$$

where $T_{i j}$ is an integration tensor independent of $x^{m}$. Now it follows from the very definition of partial differentiation that for any function $\phi\left(x^{k}, x^{m}\right)$ of $x^{k}$ and $x^{m}$, 


$$
\left[\partial_{i} \phi\left(x^{k}, x^{m}\right)\right]_{x^{m}=x_{0}^{m}}=\partial_{i} \phi\left(x^{k}, x_{0}^{m}\right) .
$$

Therefore, in consequence of (4.9), we have $\left(\rho_{, i}\right)_{x^{m}=x_{0}^{m}}=0,\left(\rho_{, i j}\right)_{x^{m}=x_{0}^{m}}=0$, and whence, if we put $x^{m}=x_{0}^{m}$ in (4.21), the result is $T_{i j} \sim g_{i j}$. This shows that (4.21) and hence also (4.20) are equivalent to (4.11). The proof of our theorem has thus been completed.

Added in proof. In connection with Theorem 4.2' I may mention that in a forthcoming paper of mine [17] a necessary and sufficient condition is obtained for a $V_{n}$ to be imbeddable in an $E_{n+1}$ as a member of $\infty^{1}$ totally umbilical hypersurfaces. There Theorem $4.2^{\prime}$ appears as a corollary to a more general result, and all the conformal-Euclidean $V_{n}$ 's which satisfy this condition of imbeddability are determined.

\section{Properly CONFormally SEPARABLE TENSORS OF THE TYPE $(n>1, m-n>1)$}

5.1. An auxiliary theorem. In this section we shall consider the conformally separable tensor

$$
{ }^{*} g_{\alpha \beta}=\left[\begin{array}{cc}
\rho^{-2} g_{i j} & 0 \\
0 & \sigma^{-2} g_{p q}
\end{array}\right] \text {, }
$$

$$
\begin{array}{r}
\alpha, \beta, \gamma, \delta=1, \cdots, m, \\
i, j, k, l=1, \cdots, n, \\
p, q, r, s=n+1, \cdots, m,
\end{array}
$$

where

$$
g_{i j}=g_{i j}\left(x^{k}\right), \quad g_{p q}=g_{p q}\left(x^{r}\right) ; \quad \rho=\rho\left(x^{\alpha}\right), \quad \sigma=\sigma\left(x^{\alpha}\right), \quad \partial_{p} \rho \neq 0, \quad \partial_{i} \sigma \neq 0 .
$$

For (5.1), we have (cf. (3.6))

$$
\begin{aligned}
-{ }^{*} R_{i p}= & (n-1) \partial_{i} \rho_{p}+(m-n-1) \partial_{p} \sigma_{i}+(m-2) \rho_{p} \sigma_{i,} \\
{ }^{*} R_{i j}= & { }^{\prime} R_{i j}+(m-n) \sigma\left(\frac{1}{\sigma}\right)_{i i j} \\
& \quad+{ }^{*} g_{i j}{ }^{*} g^{p q}\left[\rho\left(\frac{1}{\rho}\right)_{i p q}+(n-1) \rho^{2}\left(\frac{1}{\rho}\right)_{i p}\left(\frac{1}{\rho}\right)_{; q}\right] \\
{ }^{*} R_{p q}= & { }^{\prime} R_{p q}+n \rho\left(\frac{1}{\rho}\right)_{i p q} \\
& \quad+{ }^{*} g_{p q}{ }^{*} g^{i j}\left[\sigma\left(\frac{1}{\sigma}\right)_{i i j}+(m-n-1) \sigma^{2}\left(\frac{1}{\sigma}\right)_{i i}\left(\frac{1}{\sigma}\right)_{; j}\right],
\end{aligned}
$$

where the signs $\left(_{i}\right)(')$ indicate, respectively, the covariant differentiation and the Ricci tensor referred to ${ }^{*} g_{i j}$ or ${ }^{*} g_{p q}$. We suppose as usual that

$$
\rho\left(x^{k}, x_{\theta}^{r}\right)=1, \quad \sigma\left(x_{0}^{k}, x^{r}\right)=1 .
$$


To establish our main result, Theorem 5.2, in the latter part of this section, we need the following auxiliary

THEOREM 5.1. For the properly conformally separable tensor (5.1) of the type $(n>1, m-n>1)$ with (5.3) satisfied:

(i) The system of equations

$$
-{ }^{*} R_{i p}=(n-1) \partial_{i} \rho_{p}+(m-n-1) \partial_{p} \sigma_{i}+(m-2) \rho_{p} \sigma_{i}=0
$$

is equivalent to

$$
\begin{gathered}
\rho=\rho(y, z), \quad \sigma=\sigma(y, z), \\
\rho\left(y, z_{0}\right)=1, \quad \sigma\left(y_{0}, z\right)=1, \\
(n-1) \partial_{y} \rho_{z}+(m-n-1) \partial_{z} \sigma_{y}+(m-2) \rho_{z} \sigma_{y}=0\left({ }^{14}\right),
\end{gathered}
$$

where $y=y\left(x^{k}\right), z=z\left(x^{r}\right)$ are any functions of the arguments indicated, and $y_{0}=y\left(x_{0}^{\mathbf{k}}\right), z_{0}=z\left(x_{0}^{z}\right)$.

(ii) If the tensors $g_{i j}$ and $g_{p q}$ are considered as given, the following system of equations in the unknown functions $\rho\left(x^{\alpha}\right)$ and $\sigma\left(x^{\alpha}\right)$ :

$$
\begin{gathered}
{ }^{*} R_{i p}=0, \\
{ }^{*} R_{i j}-{ }^{\prime} R_{i j} \sim{ }^{*} g_{i j}, \quad{ }^{*} R_{p q}-{ }^{\prime} R_{p q} \sim{ }^{*} g_{p q}
\end{gathered}
$$

is equivalent to the system of equations consisting of $\left(5.4^{\prime}\right)$ and

$$
\begin{aligned}
\left(\frac{\rho}{\sigma}\right)^{2} \partial_{y} \sigma & =Q(z), & \left(\frac{\sigma}{\rho}\right)^{2} \partial_{\varepsilon \rho}= & J(y), \\
y_{, i j} & \sim g_{i j}, & z_{, p q} & \sim g_{p q},
\end{aligned}
$$

in the unknown functions $y=y\left(x^{k}\right), z=z\left(x^{r}\right), \rho=\rho(y, z), \sigma=\sigma(y, z)$, where $J$ and $Q$ are any functions of the arguments indicated.

5.2. Proof of Theorem 5.1 (i). Equation (5.4) can be written

$$
-(m-2) \rho_{p} \sigma_{i}=\partial_{p} \partial_{i}[(n-1) \log \rho+(m-n-1) \log \sigma] \text {. }
$$

Differentiating this partially with respect to $x^{q}$ and then taking the alternation in the indices $p$ and $q$, we $\operatorname{get}\left({ }^{15}\right)$

$$
-(m-2)\left(\sigma_{i} \partial_{[q} \rho_{p]}+\rho_{[p} \partial_{q]} \sigma_{i}\right)=0,
$$

that is,

$$
\rho\left[p \partial_{q]} \sigma_{i}=0 .\right.
$$

When the value of $\partial_{q} \sigma_{i}$ from (5.4) is substituted (which is possible because $m-n>1)$, this becomes

(14) Here we write $\partial_{y} \equiv \partial / \partial y, \partial_{z} \equiv \partial / \partial z, \rho_{z} \equiv \partial_{z} \log \rho_{,} \sigma_{y} \equiv \partial_{y} \log \sigma$.

(1b) We write, for example, $\partial_{\left[q \rho_{p]}\right.}=\partial_{q} \rho_{p}-\partial_{p} \rho_{q}$. 


$$
-\rho_{[p}\left[(n-1) \partial_{q]} \rho_{i}+(m-2) \rho_{q]} \sigma_{i}\right]=0,
$$

which, since $n>1$, reduces to

$$
\rho\left[p \partial_{q]} \rho_{i}=0 .\right.
$$

This shows that a function $\theta\left(x^{k}, x^{r}\right)$ exists such that

$$
\partial_{i} \rho_{p}=\partial_{p} \rho_{i}=\left(\partial_{i} \log \theta\right) \rho_{p}
$$

and hence

$$
\rho_{p}=\theta\left(x^{k}, x^{r}\right) w_{p}\left(x^{r}\right),
$$

where $w_{p}\left(x^{r}\right)$ are $m-n$ functions of $x^{r}$ alone. Now from the very definition of partial derivative, we have that for any fixed values $x_{1}^{\alpha}$ of $x^{\alpha}$,

$$
\partial_{p} \log \rho\left(x_{1}^{k}, x^{r}\right)=\left[\partial_{p} \log \rho\left(x^{k}, x^{r}\right)\right]_{x^{k}=x_{1}^{k}}
$$

Therefore, if we write $z \equiv \rho\left(x_{1}^{k}, x^{r}\right)$ and remember that $\rho_{p}=\partial_{p} \log \rho$, then it follows from the two preceding equations that

that is,

$$
\partial_{p} \log z=\theta\left(x_{1}^{k}, x^{r}\right) w_{p}\left(x^{r}\right)=\frac{\theta\left(x_{1}^{k}, x^{r}\right)}{\theta\left(x^{k}, x^{r}\right)} \rho_{p},
$$

$$
\partial_{p} \log \rho=\frac{\dot{\theta}\left(x^{k}, x^{r}\right)}{\theta\left(x_{1}^{k}, x^{r}\right)} \partial_{p} \log z,
$$

which shows that $\rho$ can be expressed in terms of $x^{k}$ and $z$ alone; thus,

$$
\rho=\rho\left(x^{k}, z\right) \text {. }
$$

Since (5.4) as well as the hypothesis following (5.1) remain the same when $\rho, \sigma ; n ; m-n ; i, p$ are interchanged, we have, by symmetry,

$$
\sigma=\sigma\left(y, x^{r}\right)
$$

where $y$ is defined by $y \equiv \sigma\left(x^{k}, x_{1}^{r}\right)$.

From (5.7) we have

$$
\rho_{p}=\rho_{z} \partial_{p} z, \quad \partial_{i} \rho_{p}=\left(\partial_{i} \rho_{z}\right) \partial_{p} z ; \quad \sigma_{i}=\sigma_{y} \partial_{i} y, \quad \partial_{p} \sigma_{i}=\left(\partial_{p} \sigma_{y}\right) \partial_{i} y,
$$

where $\rho_{z}=\partial_{z} \log \rho, \sigma_{y}=\partial_{y} \log \sigma$. Using these in

$$
\sigma_{[i} \partial_{j]} \rho_{p}=0 \text {, }
$$

which is the symmetric expression of $(5.6)_{a}$, we find

$$
\sigma_{y}\left(\partial_{p} z\right)\left(\partial_{[i} y\right) \partial_{j]} \rho_{s}=0 .
$$

Since by hypothesis $\partial_{p} \rho \neq 0, \partial_{i} \sigma \neq 0$, so that $\sigma_{\vartheta} \partial_{p} z \neq 0$, the above equations are equivalent to 


$$
\left(\partial_{[i} y\right) \partial_{j]} \rho_{z}=0 .
$$

From this it follows that $\rho_{z}$ can be expressed in terms of $x^{r}$ and $y$ alone. But on the other hand, (5.7) $)_{a}$ shows that $\rho_{z}$ can be expressed in terms of $x^{k}$ and $z$ alone. Therefore $\rho_{z}$ is a function of $y$ and $z$ alone, and hence $\rho$ must be of the form

$$
\rho=F\left(x^{k}\right) \phi(y, z) .
$$

Taking (5.3) into account, we have

$$
\rho=\frac{\rho\left(x^{k}, x^{r}\right)}{\rho\left(x^{k}, x_{0}^{r}\right)}=\frac{F\left(x^{k}\right) \phi(y, z)}{F\left(x^{k}\right) \phi\left(y, z_{0}\right)},
$$

where $z_{0}=z\left(x_{0}^{\gamma}\right)$. Thus

$$
\rho=\rho(y, z), \quad \rho\left(y, z_{0}\right)=1
$$

and by symmetry,

$$
\sigma=\sigma(y, z), \quad \sigma\left(y_{0}, z\right)=1 .
$$

Now for $\rho$ and $\sigma$ of the form (5.8), equations (5.4) become, after omitting the non-vanishing factor $\left(\partial_{i} y\right)\left(\partial_{p} z\right)$,

$$
(n-1) \partial_{y} \rho_{z}+(m-n-1) \partial_{z} \sigma_{\nu}+(m-2) \rho_{z} \sigma_{y}=0 .
$$

Equations (5.8) and (5.9) are identical with (5.4'), and therefore Theorem 5.1 (i) is proved.

Remark. For any $V_{m}$ with fundamental tensor $(5.1),{ }^{*} R_{i p}=0$ is the condition that there be $n$ independent congruences of Ricci curves of $V_{m}$ lying in the subspaces $x^{r}=$ const. Hence from (5.8) we have incidentally:

If, in a $V_{m}$ with a properly conformally separable fundamental tensor of the type $(n>1, m-n>1)$, the subspaces $x^{r}=$ const. contain $n$ independent congruences of Ricci curves of $V_{m}$, then the $\infty^{m-n}$ subspaces $x^{r}=$ const. and the $\infty^{n}$ subspaces $x^{k}=$ const. consist of $\infty^{1}$ families of $\infty^{m-n-1}$ isometric $V_{n}$ 's and $\infty^{1}$ families of $\infty^{n-1}$ isometric $V_{m-n}$ 's, respectively.

5.3. Proof of Theorem 5.1 (ii). By Theorem 5.1 (i), equation (5.5) which is identical with (5.4), is equivalent to $\left(5.4^{\prime}\right)$. If

$$
y=y\left({ }^{\prime} y\right), \quad z=z\left({ }^{\prime} z\right)
$$

is any nonsingular transformation from $y, z$ to ${ }^{\prime} y,{ }^{\prime} z$, then $\left(5.4^{\prime}\right)$ become

$$
\begin{gathered}
\rho=\rho\left[y\left({ }^{\prime} y\right), z\left({ }^{\prime} z\right)\right], \quad \sigma=\sigma\left[y\left({ }^{\prime} y\right), z\left({ }^{\prime} z\right)\right], \\
\rho\left[y\left({ }^{\prime} y\right), z\left({ }^{\prime} z_{0}\right)\right]=1, \quad \sigma\left[y\left({ }^{\prime} y_{0}\right), z\left({ }^{\prime} z\right)\right]=1, \\
(n-1) \partial_{y} \rho_{\prime}{ }_{z}+(m-n-1) \partial_{\prime_{z}} \sigma_{\prime} y+(m-2) \rho_{\prime_{z}} \sigma_{\prime} y=0,
\end{gathered}
$$

where ' $y_{0},{ }^{\prime} z_{0}$ are any roots of the equations $y\left({ }^{\prime} y\right)=y_{0}, z\left({ }^{\prime} z\right)=z_{0}$. Hence 
Theorem 5.1(ii) is true if a suitable nonsingular transformation $y=y\left({ }^{\prime} y\right)$, $z=z(' z)$ exists such that equations $(5.5)_{2}$ reduce to

$$
\begin{aligned}
\left(\frac{\rho}{\sigma}\right)^{2} \partial_{, y} \sigma & ={ }^{\prime} Q\left({ }^{\prime} z\right), & \left(\frac{\sigma}{\rho}\right)^{2} \partial_{{ }_{z} \rho} & ={ }^{\prime} J\left({ }^{\prime} y\right), \\
{ }^{\prime} y_{, i j} & \sim g_{i j}, & { }^{\prime}{ }_{, p q} & \sim g_{p q \cdot} .
\end{aligned}
$$

We now proceed to prove that this is the case.

By $(5.2)_{2}$, equation $(5.5)_{2 \mathrm{a}}$ is equivalent to

$\left(5.5^{\prime \prime \prime}\right)_{2 \mathrm{a}}$

$$
\left(\frac{1}{\sigma}\right)_{i i j} \sim g_{i j}
$$

When the covariant derivative $(1 / \sigma)_{; i j}$ taken with respect to ${ }^{*} g_{i j}=\rho^{-2} g_{i j}$ is expressed in terms of the covariant derivative $(1 / \sigma)_{, i}$, taken with respect to $g_{i j}$, equation $\left(5.5^{\prime \prime \prime}\right)_{2 \mathrm{a}}$ becomes (cf. (2.13))

$$
\left(\frac{1}{\sigma}\right)_{, i j}+\rho_{i}\left(\frac{1}{\sigma}\right)_{, j}+\rho_{j}\left(\frac{1}{\sigma}\right)_{, i} \sim g_{i j}
$$

By $\left(5.4^{\prime}\right)_{1}, \rho$ and $\sigma$ are functions of $y$ and $z$, and therefore

$$
\begin{aligned}
\left(\frac{1}{\sigma}\right)_{, i} & =-\frac{\partial_{y} \sigma}{\sigma^{2}} y_{, i}, \\
\left(\frac{1}{\sigma}\right)_{, i j} & =-\frac{\partial_{y} \sigma}{\sigma^{2}} y_{, i j}+2 \frac{\left(\partial_{y} \sigma\right)^{2}}{\sigma^{3}} y_{, i} y_{, i}-\frac{\partial_{y} \partial_{y} \sigma}{\sigma^{2}} y_{, i} y_{, j}, \\
\rho_{i} & =\frac{\partial_{y} \rho}{\rho} y_{, i} .
\end{aligned}
$$

When these are used in $\left(5.5^{\mathrm{iv}}\right)_{2 \mathrm{a}}$, the latter becomes

$$
-\frac{\partial_{y} \sigma}{\sigma^{2}} y_{, i j}+\left[2 \frac{\left(\partial_{y} \sigma\right)^{2}}{\sigma^{3}}-\frac{\partial_{y} \partial_{y} \sigma}{\sigma^{2}}-2 \frac{\partial_{y} \sigma}{\sigma^{2}} \frac{\partial_{y} \rho}{\rho}\right] y_{, i} y_{, j} \sim g_{i j},
$$

which, because $\partial_{y} \sigma \neq 0$ by hypothesis, can be written

$$
y_{, i j}+\left(\partial_{y} \log \frac{\rho^{2} \partial_{y} \sigma}{\sigma^{2}}\right) y_{, i} y_{, j} \sim g_{i j}
$$

A consequence of this equation is

$$
\partial_{y} \log \frac{\rho^{2} \partial_{y} \sigma}{\sigma^{2}}=\text { a function of } y \text { alone. }
$$

For, if we write (5.10) as

$$
y_{, i j}+\lambda(y, z) y_{, i} y_{, j} \sim g_{i j},
$$


and eliminate $y_{, i j}$ from it and

$$
y_{, i j}+\lambda\left(y, z_{1}\right) y_{, i} y_{, j} \sim g_{i j},
$$

where $z_{1}$ is a constant, we find

$$
\left[\lambda(y, z)-\lambda\left(y, z_{1}\right)\right] y_{, i} y_{, i} \sim g_{i j} .
$$

Since $g_{i j}$ is of rank greater than 1 , the coefficient of $y_{. i} y_{, j}$ in the above equation must be zero; thus $\lambda(y, z)=\lambda\left(y, z_{1}\right)$, which proves (5.11).

This being the case, we have by integration of (5.11) that

$$
\frac{\rho^{2} \partial_{y} \sigma}{\sigma^{2}}=I(y) Q(z) \text {. }
$$

In like manner we derive from $(5.5)_{2 b}$ that

$$
\frac{\sigma^{2} \partial_{z} \rho}{\rho^{2}}=J(y) P(z) .
$$

Here $I, J, P, Q$ are some functions of the arguments indicated.

Now consider the functions ' $y,{ }^{\prime} z$ introduced (to within integration constants) by

$$
{ }^{\prime} y=\int I(y) d y, \quad{ }^{\prime} z=\int P(z) d z .
$$

Since $\partial_{z} \rho \neq 0, \partial_{y} \sigma \neq 0$ by hypothesis, it follows from (5.12) that neither $I(y)$ nor $P(z)$ can be identically zero. Consequently, (5.13) define a nonsingular transformation, which evidently carries $(5.12)$ into

$$
\frac{\rho^{2} \partial_{{ }^{\prime}} \sigma}{\sigma^{2}}={ }^{\prime} Q\left({ }^{\prime} z\right), \quad: \quad \frac{\sigma^{2} \partial_{z} \rho}{\rho^{2}}={ }^{\prime} J\left({ }^{\prime} y\right) .
$$

Finally, if we recall the way in which (5.10) was derived from $(5.5)_{2 a}$, it will at once become obvious that the expression for (5.5) $2 \mathrm{a}$ in terms of ' $y$ and ' $z$ is obtained by replacing $y$ by ' $y$ in (5.10); that is, (5.13) transforms (5.5) $2 \mathrm{a}$ in to

$$
{ }^{\prime} y_{, i i}+\left(\partial_{, y} \log \frac{\rho^{2} \partial_{{ }^{\prime}} \sigma}{\sigma^{2}}\right){ }^{\prime} y_{, i}{ }^{\prime} y_{, j} \sim g_{i j} .
$$

In consequence of $(5.14)_{1 \mathrm{a}}$, this becomes

$$
' y_{, i j} \sim g_{i j} .
$$

Similarly, in terms of ' $y$ and ' $z$, equation $(5.5)_{2 \mathrm{~b}}$ becomes

$$
\left.{ }^{\prime} z, p q\right) g_{p q \cdot}
$$

Equations (5.14) are identical with $\left(5.5^{\prime \prime}\right)_{2}$, which proves our theorem. 
Remark. From the above proof, it is easily seen that equations $(5.5)_{1}$, $(5.5)_{2 \mathrm{a}}$, by themselves, are equivalent to $\left(5.4^{\prime}\right),\left(5.5^{\prime}\right)_{1 \mathrm{a}},\left(5.5^{\prime}\right)_{2 \mathrm{a}}$; and equations $(5.5)_{1},(5.5)_{2 \mathrm{~b}}$, by themselves, to $\left(5.4^{\prime}\right),\left(5.5^{\prime}\right)_{1 \mathrm{~b}},\left(5.5^{\prime}\right)_{2 \mathrm{~b}}$.

5.4 An important property. We are now ready to prove the following

THEOREM 5.2. If a properly conformally separable tensor ${ }^{*} g_{\alpha \beta}$ of the type $(n, m-n)$ represents an $E_{m}$ and each of its component tensors either is of dimension 2 or represents $E_{n}$ 's, then ${ }^{*} g_{\alpha \beta}$ is conformal to a separable tensor of the type $(n, m-n)$.

Proof. By supposition, we have

$$
\begin{aligned}
& { }^{*} R_{\alpha \beta}=-(m-1) c{ }^{*} g_{\alpha \beta}, \\
& c=\text { const. }, \\
& { }^{\prime} R_{i j}=-(n-1) * a\left(x^{k}, x^{r}\right) * g_{i j}, \\
& { }^{\prime} R_{p q}=-(m-n-1) * b\left(x^{k}, x^{r}\right)^{*} g_{p q} .
\end{aligned}
$$

As a consequence of these equations, equations (5.5) are true. Thus by Theorem 5.1 (ii), two functions $y=y\left(x^{k}\right), z=z\left(x^{r}\right)$ exist such that the following equations are satisfied:

$$
\left\{\begin{array}{c}
\rho=\rho(y, z), \quad \sigma=\sigma(y, z), \\
\rho\left(y, z_{0}\right)=1, \quad \sigma\left(y_{0}, z\right)=1, \\
(n-1) \partial_{y} \rho_{z}+(m-n-1) \partial_{z} \sigma_{y}+(m-2) \rho_{z} \sigma_{y}=0 .
\end{array}\right.
$$

$$
\left\{\begin{aligned}
\left(\frac{\rho}{\sigma}\right)^{2} \partial_{y} \sigma & =Q(z), & \left(\frac{\sigma}{\rho}\right)^{2} \partial_{z} \rho & =J(y), \\
y_{, i j} & \sim g_{i j}, & z_{, p q} & \sim g_{p q} .
\end{aligned}\right.
$$

Our theorem will be proved if we can show that as a result of (5.16), (5.17) and (5.18), the function $\rho / \sigma$ is of the form ' $\rho(y) /{ }^{\prime} \sigma(z)$. We treat the two cases $n>2, m-n>1$ and $n=m-n=2$ separately.

Case 1. $n>2, m-n>1$.

Since $n>2$, we have by supposition that $\rho^{-2} g_{i j}$ represents $E_{n}$ 's. Therefore it follows from $(5.16)_{\mathrm{a}}$ and $(5.17)_{1 \mathrm{a}}$ that ${ }^{*} a\left(x^{k}, x^{r}\right)={ }^{*} a(z)$. On account of $(5.17)_{2 \mathrm{a}}, g_{i j}$ is the fundamental tensor of an $E_{n}$, whose scalar curvature is $a={ }^{*} a\left(z_{0}\right)$. Thus, by Theorem 2.1, equation $(5.16)_{\mathrm{a}}$ implies that

$$
\rho_{, i i} \sim g_{i j} .
$$

In virtue of $(5.17)_{1 \mathrm{a}}$, this can be written

$$
\left(\partial_{y} \rho\right) y_{, i j}+\left(\partial_{y} \partial_{y} \rho\right) y_{, i} y_{, j} \sim g_{i j}
$$

which becomes, because of $(5.18)_{2 \mathrm{a}}$,

$$
\left(\partial_{y} \partial_{y} \rho\right) y_{, i} y_{, i} \sim g_{i j} .
$$


Since $g_{i j}$ is of rank greater than 1 , it follows from this that $\partial_{y} \partial_{y} \rho=0$, whence (5.20)

$$
\rho=U(z) y+V(z) \text {. }
$$

Using this in $(5.18)_{1 \mathrm{a}}$ we have

$$
\partial_{\nu}\left(\frac{1}{\sigma}\right)=-Q(U y+V)^{-2}= \begin{cases}-Q V^{-2} & \text { if } U=0 \\ \frac{Q}{U} \partial_{y}(U y+V)^{-1} & \text { if } U \neq 0 .\end{cases}
$$

Integrating these with respect to $y$ and then making use of (5.20), we find that for both cases $\rho / \sigma$ is of the form

$$
\frac{\rho}{\sigma}=W(z) y+Z(z)
$$

The $U, V$ in (5.20) and $W, Z$ in (5.21) are all functions of $z$ alone.

If either $W$ or $Z$ is identically zero, $\rho / \sigma$ will be of the form ' $\rho(y) /{ }^{\prime} \sigma(z)$, and our theorem is proved.

Now suppose that neither $W$ nor $Z$ is identically zero. When (5.20), (5.21) are used in $(5.17)_{3}$, the latter reduces to

$$
\begin{aligned}
& (m-2)\left(U^{\prime} Z-V^{\prime} W\right)(W y+Z) \\
& +(m-n-1)\left(W Z^{\prime}-W^{\prime} Z\right)(U y+V)=0,
\end{aligned}
$$

where the prime denotes differentiation. From this it can be proved that

$$
W Z^{\prime}-W^{\prime} Z=0 \text {. }
$$

Assume that this is not true. Then since $W \neq 0, Z \neq 0$, we have from (5.22) that

$$
U / W=V / Z=X(z),
$$

where $X \neq 0$, otherwise, $\rho=0$ by (5.20). Now it is easily verified that in consequence of the above equations, $(5.22)$ becomes $(n-1)\left(W Z^{\prime}-W^{\prime} Z\right) X=0$, which cannot be satisfied. Thus (5.23) is true, and consequently $W$, and $Z$ differ by a constant factor. Hence it follows from (5.21) that $\rho / \sigma$ is the form ${ }^{\prime} \rho(y) /{ }^{\prime} \sigma(z)$, and the proof of Theorem 5.2 for the case $n>2, m-n>1$ is completed.

Remark. We observe that in the above proof we made use only of equations $(5.16)_{\mathrm{a}},(5.17),(5.18)_{1 \mathrm{a}},(5.18)_{2 \mathrm{a}}$, but not of $(5.15),(5.16)_{\mathrm{b}},(5.18)_{1 \mathrm{~b}}$, $(5.18)_{2 \mathrm{~b}}$. Now by the remark at the end of $\$ 5.3$, equations $(5.17),(5.18)_{1 \mathrm{a}}$, $(5.18)_{2 \mathrm{a}}$ are equivalent to $(5.5)_{1},(5.5)_{2 \mathrm{a}}$, namely,

$$
{ }^{*} R_{i p}=0, \quad * R_{i j}-{ }^{\prime} R_{i j} \sim{ }^{*} g_{i j} \text {. }
$$

Thus our conclusion that $\rho / \sigma$ is of the form $\rho(y) /{ }^{\prime} \sigma(z)$ is in fact a conse- 
quence of the following equations:

$$
{ }^{*} R_{i p}=0, \quad ' R_{i j} \sim *^{*} g_{i j}, \quad{ }^{*} R_{i j} \sim *^{*} g_{i j}
$$

Hence:

If, at each point $P$, coordinates $x_{1}^{\alpha}$, of $a V_{m}$ with properly conformally separable fundamental tensor ${ }^{*} g_{\alpha \beta}$ of the type $(n>2, m-n>1)$, every direction in the $V_{n}: x^{r}=x_{1}^{r}$ is a Ricci direction both of $V_{m}$ and of $V_{n}$, then ${ }^{*} g_{\alpha \beta}$ is conformal to a separable tensor of the type $(n, m-n)$.

Case 2. $n=m-n=2$.

In this case, equation (5.19) is in general not true, and we shall base our proof of Theorem 5.2 on (5.17) and (5.18) alone. Equation (5.17) 8 is now equivalent to

$$
\partial_{y} \rho_{z}+\rho_{z} \sigma_{y}=\omega, \quad \partial_{z} \sigma_{y}+\rho_{z} \sigma_{y}=-\omega,
$$

where $\omega=\omega(y, z)$. If $\omega=0$, then since $\rho_{z} \neq 0,(5.24)_{\mathrm{a}}$ can be written

$$
\partial_{y} \log \left(\sigma \rho_{z}\right)=0,
$$

which gives us, on integration,

$$
\sigma \rho_{z}=\frac{1}{\sigma \sigma(z)}, \text { that is, } \quad \frac{\sigma}{\rho} \partial_{z} \rho=\frac{1}{\sigma(z)} .
$$

Comparison of the last equation with $(5.18)_{1 \mathrm{~b}}$ shows that $\sigma / \rho={ }^{\prime} \sigma(z) J(y)$, which proves our theorem.

We now suppose that $\omega \neq 0$ and always bear in mind that $J=J(y), Q=Q(z)$. Then on account of $(5.18)_{1}$, equation $(5.24)_{a}$ can be written

that is,

$$
\omega=\frac{J \rho}{\sigma^{2}} \partial_{y} \log \left(J \frac{\rho}{\sigma}\right)=\frac{J \rho}{\sigma^{2}}\left(\frac{J^{\prime}}{J}+\rho_{y}-\frac{Q \sigma}{\rho^{2}}\right),
$$

$$
\partial_{\nu}\left(\frac{1}{\rho}\right)=\frac{J^{\prime}}{\rho J}-\frac{Q \sigma}{\rho^{3}}-\frac{\omega \sigma^{2}}{J \rho^{2}} .
$$

In like manner, $(5.24)_{\mathrm{b}}$ can be reduced to

$$
\frac{Q \partial_{z} \sigma}{\rho^{2}}=-\frac{Q^{\prime} \sigma}{\rho^{2}}+\frac{J Q}{\rho \sigma}-\omega .
$$

We now find the integrability condition $\partial_{z} \partial_{y}(1 / \rho)=\partial_{y} \partial_{z}(1 / \rho)$ for $(5.18)_{1 b}$ and $(5.25)_{\mathrm{a}}$. Differentiate $(5.25)_{\mathrm{a}}$ with respect to $z$ and we have

$$
\begin{aligned}
\partial_{z} \partial_{\nu}\left(\frac{1}{\rho}\right)=\frac{J^{\prime}}{J} \partial_{z}\left(\frac{1}{\rho}\right)-\left[\frac{Q^{\prime} \sigma}{\rho^{3}}+\frac{Q \partial_{z} \sigma}{\rho^{3}}+\frac{3 Q \sigma}{\rho^{2}} \partial_{z}\left(\frac{1}{\rho}\right)\right] \\
-\frac{1}{J}\left[\frac{\sigma^{2}}{\rho^{2}} \partial_{z} \omega+\frac{2 \omega \sigma \partial_{z} \sigma}{\rho^{2}}+\frac{2 \omega \sigma^{2}}{\rho} \partial_{z}\left(\frac{1}{\rho}\right)\right] .
\end{aligned}
$$


On the other hand we have from (5.18)

(5.27) $\partial_{z}\left(\frac{1}{\rho}\right)=-\frac{J}{\sigma^{2}}, \quad \partial_{y} \partial_{z}\left(\frac{1}{\rho}\right)=-\frac{J^{\prime}}{\sigma^{2}}+2 J \frac{\partial_{y} \sigma}{\sigma^{3}}=-\frac{J^{\prime}}{\sigma^{2}}+\frac{2 J Q}{\rho^{2} \sigma}$.

Using (5.27) ${ }_{1}$ and $(5.25)_{\mathrm{b}}$ in (5.26) and comparing the result with $(5.27)_{2}$, we find

$$
\begin{aligned}
-\frac{J^{\prime}}{\sigma^{2}}+\frac{2 J Q}{\rho^{2} \sigma}= & \frac{J^{\prime}}{J}\left(-\frac{J}{\sigma^{2}}\right)-\frac{Q^{\prime} \sigma}{\rho^{3}}-\left(-\frac{Q^{\prime} \sigma}{\rho^{3}}+\frac{J Q}{\rho^{2} \sigma}-\frac{\omega}{\rho}\right)-\frac{3 Q \sigma}{\rho^{2}}\left(-\frac{J}{\sigma^{2}}\right) \\
& -\frac{1}{J}\left[\frac{\sigma^{2}}{\rho^{2}} \partial_{z} \omega+2 \omega \sigma\left(-\frac{Q^{\prime} \sigma}{Q \rho^{2}}+\frac{J}{\rho \sigma}-\frac{\omega}{Q}\right)+\frac{2 \omega \sigma^{2}}{\rho}\left(-\frac{J}{\sigma^{2}}\right)\right],
\end{aligned}
$$

which simplifies into

$$
\frac{\omega}{\rho}-\frac{1}{J}\left(\frac{\sigma^{2}}{\rho^{2}} \partial_{z} \omega-\frac{2 \omega \sigma^{2} Q^{\prime}}{\rho^{2} Q}-\frac{2 \omega^{2} \sigma}{Q}\right)=0,
$$

that is,

$$
\frac{J \rho}{\sigma^{2}}-\frac{\partial_{\partial} \omega}{\omega}+\frac{2 Q^{\prime}}{Q}+\frac{2 \omega \rho^{2}}{\sigma Q}=0 .
$$

This is the integrability condition we wished to establish. But because of $(5.18)_{1},(5.24)_{b}$, we have

$$
\frac{J \rho}{\sigma^{2}}=\frac{\partial_{s} \rho}{\rho}, \quad \frac{\omega \rho^{2}}{\sigma Q}=\frac{\omega}{\sigma_{y}}=-\partial_{z} \log \left(\rho \sigma_{y}\right) .
$$

Therefore (5.28) becomes

$$
\partial_{z} \log \left[\frac{\rho}{\omega}\left(\frac{Q}{\rho \sigma_{y}}\right)^{2}\right]=0,
$$

which, by $(5.18)_{1 \mathrm{a}}$, can be written

$$
\partial_{s} \log \frac{\rho^{3}}{\omega \sigma^{2}}=0 .
$$

Following a procedure symmetric to the above one, we can prove that

$$
\partial_{y} \log \frac{\sigma^{3}}{\omega \rho^{2}}=0
$$

From (5.29) it follows at once that

$$
\partial_{y} \partial_{z} \log \frac{\rho}{\sigma}=0
$$


which show that $\rho / \sigma$ is of the form ${ }^{\prime} \rho(y) /{ }^{\prime} \sigma(z)$. Thus the proof of Theorem 5.2 is completed.

Remark. The above proof for the case $n=m-n=2$ holds also for the more general case $n=m-n>1$, but not for other cases. Indeed, when $n>1$, $m-n>1$, equation (5.17) 3 may be replaced by (cf. (5.24))

$$
\partial_{y} \rho_{z}+\rho_{z} \sigma_{y}=\frac{\omega}{n-1}, \quad \partial_{z} \sigma_{y}+\rho_{z} \sigma_{y}=-\frac{\omega}{m-n-1},
$$

and if we carry out on these equations a procedure similar to that which we did on (5.24) for the case $\omega \neq 0$, then the final result corresponding to (5.30) is

$$
\partial_{y} \partial_{z}\left[\left(\frac{n-1}{m-n-1}+4\right) \log \rho-\left(\frac{m-n-1}{n-1}+4\right) \log \sigma\right]=0 .
$$

This reduces to (5.30) when and only when $n=m-n$, which proves our assertion. Moreover, since our proof depends only on (5.17), (5.18), which are equivalent to (5.5), we have

If the fundamental tensor ${ }^{*} g_{\alpha \beta}$ of a $V_{2 n}(n>1)$ is a properly conformally separable tensor of the type $(n, n)$ and the Ricci tensors ${ }^{*} R_{\alpha \beta},{ }^{\prime} R_{i j},{ }^{\prime} R_{p q}$ of $V_{2 n}$ and its subspaces $x^{r}=$ const. and $x^{k}=$ const. satisfy the relations:

$$
{ }^{*} R_{i p}=0, \quad{ }^{*} R_{i j}-{ }^{\prime} R_{i j} \sim{ }^{*} g_{i j}, \quad{ }^{*} R_{p q}-{ }^{\prime} R_{p q} \sim{ }^{*} g_{p q},
$$

then ${ }^{*} g_{\alpha \beta}$ is conformal to a separable tensor of the type $(n, n)$.

6.1. Main results. Theorem 5.2 enables us to bring to a satisfactory conclusion our study of a properly conformally separable tensor which represents an $E_{m}$ and each of whose component tensors either is of dimension 2 or represents $E$ 's.

Consider the properly conformally separable tensor

$$
* g_{\alpha \beta}=\tau^{-2 \prime \prime} g_{\alpha \beta} \equiv \tau^{-2}\left[\begin{array}{cc}
\bar{g}_{i j} & 0 \\
0 & \bar{g}_{p q}
\end{array}\right] \text {, }
$$

$$
\begin{array}{r}
\alpha, \beta, \gamma, \epsilon=1, \cdots, m, \\
i, j, k, l=1, \cdots, n, \\
p, q, r, s=n+1, \cdots, m,
\end{array}
$$

where

$$
\begin{gathered}
n>1, \quad m-n>1, \\
\tau=\tau\left(x^{\alpha}\right), \quad \partial_{i} \tau \neq 0, \quad \partial_{p} \tau \neq 0, \\
\bar{g}_{i j}=\bar{g}_{i j}\left(x^{k}\right), \quad \bar{g}_{p q}=\bar{g}_{p q}\left(x^{r}\right) .
\end{gathered}
$$

Let quantities referred to ${ }^{\prime \prime} g_{\alpha \beta}, \bar{g}_{i j}$, or $\bar{g}_{p q}$ and covariant differentiations taken with respect to them be marked by the signs $\left({ }^{\prime \prime}\right),(-) ;(),.(/)$. Then we have (cf. (3.2), (3.3))

$$
{ }^{\prime \prime} \Gamma_{i j}^{k}=\Gamma_{i j}^{k}, \quad{ }^{\prime \prime} \Gamma_{p q}^{r}=\Gamma_{p q}^{r}, \quad "{ }^{\prime} \Gamma_{\alpha \beta}^{\gamma}=0
$$

if $\alpha, \beta, \gamma$ are not all in the same range; and consequently, 


$$
\begin{aligned}
& \tau_{: i p}=\partial_{p} \partial_{i} \tau, \quad \tau_{: i j}=\tau_{/ i j}, \quad \tau_{: p q}=\tau_{/ p q}, \\
& \text { " } R_{i p}=0, \quad " R_{i j}=\bar{R}_{i j}, \quad " R_{p q}=\bar{R}_{p q} \text {. }
\end{aligned}
$$

Also from (6.1) we have $[5$, p. $90(28.6)]$

$(6.5){ }^{*} R_{\alpha \beta}=" R_{\alpha \beta}-(m-2) \frac{\tau_{: \alpha \beta}}{\tau}+" g_{\alpha \beta} " g^{\gamma \epsilon}\left[-\frac{\tau_{: \gamma \epsilon}}{\tau}+(m-1) \frac{\tau_{: \gamma \gamma} \tau_{: \epsilon}}{\tau^{2}}\right]$.

Now suppose that (6.1) represents an $E_{m}(c)$, that is, that

$$
{ }^{*} R_{\alpha \beta}=-(m-1) c^{*} g_{\alpha \beta},
$$$$
c=\text { const. }
$$

In consequence of this and equations (6.1)-(6.4), equation $(6.5)$ for $(\alpha, \beta)$ $=(i, p)$ becomes $\partial_{p} \partial_{i} \tau=0$, and therefore $\left({ }^{16}\right)$

$$
\tau=y\left(x^{k}\right)+z\left(x^{r}\right),
$$

where $y \neq$ const., $z \neq$ const. because of (6.2). Hence (6.1) may be written

$$
*_{\alpha \beta}=(y+z)^{-2}\left[\begin{array}{cc}
\bar{g}_{i j} & 0 \\
0 & \bar{g}_{p q}
\end{array}\right] \equiv(y+z)^{-2}\left[\begin{array}{cc}
y^{2} g_{i j} & 0 \\
0 & z^{2} g_{p q}
\end{array}\right] .
$$

If $n>2$ and the component tensor ${ }^{*} g_{i j}=[(y+z) / y]^{-2} g_{i j}$ represents $E_{n}$ 's $\left(S_{n}\right.$ 's), then $g_{i j}$ represents an $E_{n}\left(S_{n}\right)$, because ${ }^{*} g_{i j}$ becomes $g_{i j}$ for $z=0$. Consequently, we have by Theorem 2.1

$$
\left(\frac{y+z}{y}\right)_{, i j} \sim g_{i j}, \text { that is, }\left(\frac{1}{y}\right)_{, i j} \sim g_{i j},
$$

where, as usual, a comma denotes covariant differentiation with respect to $g_{i j}$. Thus by Theorems 2.1 and 2.2, the tensor $y^{2} g_{i j}=\bar{g}_{i j}$ is the fundamental tensor of an $E_{n}\left(S_{n}\right)$.

We now suppose, besides (6.7), also that each of the component tensors of the tensor (6.8) either is of dimension 2 or represents $E$ 's. Then it follows from the above observation that

$$
\begin{aligned}
& \bar{R}_{i j}=-(n-1) \bar{a}\left(x^{k}\right) \bar{g}_{i j}, \\
& \bar{R}_{p q}=-(m-n-1) b\left(x^{r}\right) \bar{g}_{p q},
\end{aligned}
$$

where $\bar{a}\left(x^{k}\right)$ is constant when $n>2$, and $b\left(x^{r}\right)$ is constant when $m-n>2$. On account of (6.3), (6.4), (6.6), (6.7), (6.9) and (6.10), equation (6.5) for $(\alpha, \beta)$ $=(i, j)$ and $(p, q)$ become

$$
\begin{aligned}
(6.11)_{\mathrm{a}} \quad-(m-1) c \tau^{-2} \bar{g}_{i j}=-(n-1) \bar{a}\left(x^{k}\right) \bar{g}_{i j}-(m-2) \tau^{-1} y_{/ i j} \\
\quad+\bar{g}_{i j}\left[-\left(\bar{g}^{h k} y_{/ h k}+\bar{g}^{r s} z_{/ r s}\right) \tau^{-1}+(m-1)\left(\bar{g}^{h k} y_{/ h} y_{/ k}+\bar{g}^{r s} z_{/ r} z / s\right) \tau^{-2}\right],
\end{aligned}
$$

(16) We note that these $y, z$ are not identical with the $y, z$ which appeared in $\$ \$ 5.1-5.4$. 
$(6.11)_{b}$

$$
\begin{aligned}
- & (m-1) c \tau^{-2} \bar{g}_{p q}=-(m-n-1) b\left(x^{r}\right) \bar{g}_{p q}-(m-2) \tau^{-1} z_{/ p q} \\
& +\bar{g}_{p q}\left[-\left(\bar{g}^{h k} y_{/ h k}+\bar{g}^{r s} z_{/ r s}\right) \tau^{-1}+(m-1)\left(\bar{g}^{h k} y_{/ h} y_{/ k}+\bar{g}^{r s} z_{/ r} z / s\right) \tau^{-2}\right] .
\end{aligned}
$$

From these it follows at once that

$$
y_{/ i j}=-L \bar{g}_{i j}, \quad z_{/ p q}=-S \bar{g}_{p q},
$$

which, because of (6.10), imply (cf. Theorems 2.3 and (2.9), (2.10)), respectively.

(6.13) $\overline{\mathrm{g}}^{i j} y_{/ i} y_{/ i}=-2 M(y), \quad L=L(y)=M^{\prime}(y), \quad \bar{a}\left(x^{k}\right)=\bar{a}(y)=M^{\prime \prime}(y)$,

$(6.13)_{\mathrm{b}} \quad \bar{g}^{p q} z_{/ p} z / q=-2 T(z), \quad S=S(z)=T^{\prime}(z), \quad b\left(x^{r}\right)=b(z)=T^{\prime \prime}(z)$,

where the prime denotes differentiation. In consequence of these, equations (6.11) are equivalent to (6.12), (6.13) and

$$
\begin{aligned}
-(m-1) c \tau^{-2} & +(n-1) M^{\prime \prime}-(m-2) M^{\prime} \tau^{-1} \\
& =-(m-1) c \tau^{-2}+(m-n-1) T^{\prime \prime}-(m-2) T^{\prime} \tau^{-1} \\
& =\left[n M^{\prime}+(m-n) T^{\prime}\right] \tau^{-1}-2(m-1)(M+T) \tau^{-2}
\end{aligned}
$$

These last equations together with (6.7) can be solved for $M(y)$ and $T(z)$. Indeed, the first equation of (6.14) can be written

$$
(m-2)\left(M^{\prime}-T^{\prime}\right)-(y+z)\left[(n-1) M^{\prime \prime}-(m-n-1) T^{\prime \prime}\right]=0 .
$$

Differentiating this partially with respect to $y$ twice, we find

$$
(m-2 n) M^{\prime \prime \prime}-(n-1)(y+z) M^{\text {iv }}=0,
$$

which, because $M$ is a function of $y$ alone, gives us $M^{\mathrm{iv}}=0$. Hence

$$
M(y)=a_{0}+a_{1} y+a_{2} y^{2}+a_{3} y^{3},
$$

and by symmetry,

$$
T(z)=b_{0}+b_{1} z+b_{2} z^{2}+b_{3} z^{3}
$$

where the $a$ 's and $b$ 's are constants. When these values of $M$ and $T$ are substituted in (6.15), the latter reduces to

$$
b_{1}=a_{1}, \quad b_{2}=-a_{2}, \quad\left\{\begin{array}{lll}
b_{3}=a_{3}=0 & \text { if } & m \neq 2 n, \\
b_{3}=a_{3} & \text { if } & m=2 n .
\end{array}\right.
$$

Now in consequence of (6.16) and (6.17), equations (6.14) become

$$
\begin{aligned}
c & =2\left(a_{0}+b_{0}\right), \\
a_{3} & =0 \text { if } m=2 n=4 \text { are not satisfied. }
\end{aligned}
$$

Thus, the solution of equations (6.14) is given by (6.16)-(6.19).

By Theorem 5.2, the preceding results prove the necessity of the condition in the following 
THEOREM 6.1. In order that a properly conformally separable tensor ${ }^{*} g_{\alpha \beta}$ of the type $(n>1, m-n>1)$ may represent an $E_{m}$ and each of its component tensors either be of dimension 2 or represent $E$ 's, it is necessary and sufficient that ${ }^{*} g_{\alpha \beta}$ be of the form (6.8) and equations (6.10), (6.12), (6.13), (6.16)-(6.19) be satisfied.

The sufficiency of the condition in this theorem can be proved as follows. If $n>2,(6.10)_{a}$ shows that $\bar{g}_{i j}$ represents an $E_{n}$, and consequently by $(6.12)_{2}$, the tensor $g_{i j}=y^{-2} \bar{g}_{i j}$ also represents an $E_{n}$ (Theorem 2.1). Thus equations (6.9) are satisfied, and accordingly the component tensor ${ }^{*} g_{i j}=[(y+z) / y]^{-2} g_{i j}$ represents $E_{n}$ 's. Similarly, it follows from $(6.10)_{\mathrm{b}}$ and $(6.12)_{\mathrm{b}}$ that if $m-n>2$, the component tensor ${ }^{*} g_{p q}$ represents $E_{m-n}$ 's. Finally, from the way in which equations (6.10), (6.12), (6.13), (6.16)-(6.19) were derived, it is readily seen that if these equations are satisfied, equation (6.6) must also be satisfied. Hence ${ }^{*} g_{\alpha \beta}$ represents an $E_{m}$ and our theorem is completely proved.

Now we are ready to establish the following three main results.

THEOREM 6.2. If a properly conformally separable tensor represenis an $E_{m}$ and one of its component tensors represents $E^{\prime} s$ or $S_{2}$ 's, then the other component tensor, if it is of dimension 2, represents $S_{2}$ 's.

TheoRem 6.3. In order that a properly conformally separable tensor ${ }^{*} g_{\alpha \beta}$ may represent an $E_{m}$ and each of its component tensors $E^{\prime}$ 's or $S_{2}$ 's it is necessary and sufficient that the following conditions be fulfilled:

(1) ${ }^{*} g_{\alpha \beta}$ is of the form

$$
(y+z)^{-2}\left[\begin{array}{cc}
\bar{g}_{i j} & 0 \\
0 & \bar{g}_{p q}
\end{array}\right], \quad \begin{aligned}
y & =y\left(x^{k}\right), \quad z=z\left(x^{r}\right), \\
\bar{g}_{i j} & =\bar{g}_{i j}\left(x^{k}\right), \quad \bar{g}_{p q}=\bar{g}_{p q}\left(x^{r}\right) .
\end{aligned}
$$

(2) The tensors $\bar{g}_{i j}, \bar{g}_{p q}$ each represent an $E$ or $S_{2}$ with scalar curvatures $\bar{a}, \bar{b}$ connected by $\bar{a}+\bar{b}=0$.

(3) The equations

$$
y_{/ i j}=-(\bar{a} y+f) \bar{g}_{i j}, \quad z_{/ p q}=-(\overline{b z}+f) \bar{g}_{p q}
$$

are satisfied with a constant $f$.

If these conditions are fulfilled, the scalar curvature $c$ of ${ }^{*} g_{\alpha \beta}$ is equal to the sum of the scalar curvatures of $y^{-2} \bar{g}_{i j}$ and $z^{-2} \bar{g}_{p q}$ (each of which, as is implied by (2) and (3), is the fundamental tensor of an $E$ or $S_{2}$ ).

THEOREM 6.4. In order that a properly conformally separable tensor ${ }^{*} g_{\alpha \beta}$ of the type $(2,2)$, whose component tensors do not represent $S_{2}$ 's, may be the fundamental tensor of an $E_{4}$, it is necessary and sufficient that $(1){ }^{*} g_{\alpha \beta}$ be of the form

$$
A(\bar{a}+\bar{b})^{-2}\left[\begin{array}{cc}
\bar{g}_{i j} & 0 \\
0 & \bar{g}_{p q}
\end{array}\right], \quad \quad A=\text { const. }
$$


and (2) the equations

$$
\bar{a}_{/ i j}=-(1 / 2)\left(\bar{a}^{2}+B\right) \bar{g}_{i j}, \quad \bar{b}_{/ p q}=-(1 / 2)\left(b^{2}+B\right) \bar{g}_{p q}
$$

be satisfied with a constant $B$, where $\bar{a}, \bar{b}$ are the scalar curvatures of the fundamental tensors $\bar{g}_{i j}, \bar{g}_{p q}$.

The proof of these theorems will be based on Theorem 6.1. For Theorem 6.2 , we suppose for the moment that $m-n=2$ and that if $n=2,{ }^{*} g_{i j}$ represents $S_{2}$ 's. Theorem 6.2 will be proved if we can show that in this case $a_{3}=0$. Indeed, if $a_{3}=0$, then by (6.17), $b_{3}=0$, and consequently, by $(6.13)_{\mathrm{b}}$ and $(6.16)_{\mathrm{b}}, \bar{b}\left(x^{r}\right)=T^{\prime \prime}(z)=2 b_{2}=$ const. Therefore $\bar{g}_{p q}$ represents an $S_{2}$. Since $(6.12)_{\mathrm{b}}$ can be written as $(y+z)_{/ p q}=-S \bar{g}_{p q}$, the component tensor ${ }^{*} g_{p q}$ $=(y+z)^{-2} \bar{g}_{p q}$ represents $S_{2}$ 's. It remains therefore to prove that $a_{3}=0$.

If $n>2, a_{3}=0$ is given by (6.19) without further proof. If $n=2$, we have by hypothesis that ${ }^{*} g_{i j}=(y+z)^{-2} \bar{g}_{i j}$ represents $S_{2}$ 's, which implies that the tensor $g_{i j} \equiv y^{-2} \bar{g}_{i j}$ also represents an $S_{2}$. Therefore, in consequence of (6.12) a, $\bar{g}_{i j}=y^{2} g_{i j}$ is the fundamental tensor of an $S_{2}$ (Theorem 2.5). Hence we have from (6.13) and (6.16) at $\bar{a}$ at $\bar{a}\left(x^{k}\right)=M^{\prime \prime}(y)=2 a_{2}+6 a_{3} y=$ const. From this it follows that $a_{3}=0$, as was to be proved. Theorem 6.1 has thus been completely established.

As a consequence of Theorem 6.2 , for a properly conformally separable tensor which represents an $E_{m}$ and each of whose component tensors either is of dimension 2 or represents $E$ 's, only two cases can happen: either (1) each of its component tensors represents $E$ 's or $S_{2}$ 's, or (2) $m=2 n=2$ and neither of them represents $S_{2}$ 's. They are the two cases which we deal with in Theorems 6.3 and 6.4. For them, we have, respectively,

$$
\begin{gathered}
M(y)=a_{0}+a_{1} y+a_{2} y^{2}, \quad T(z)=b_{0}+b_{1} z+b_{2} z^{2}, \\
a_{1}=b_{1}, \quad a_{2}+b_{2}=0 \\
M(y)=a_{0}+a_{1} y+a_{2} y^{2}+a_{3} y^{3}, \quad T(z)=b_{0}+b_{1} z+b_{2} z^{2}+b_{3} z^{3}, \\
a_{1}=b_{1}, \quad a_{2}+b_{2}=0, \quad a_{3}=b_{3} \neq 0 .
\end{gathered}
$$

Now for case (1), the scalar curvatures of the fundamental tensors $\bar{g}_{i j}$, $\bar{g}_{p q}, g_{i j}=y^{-2} \bar{g}_{i j}, g_{p q}=z^{-2} \bar{g}_{p q}$ are, respectively $\bar{a}=2 a_{2}, b=2 b_{2}, 2 a_{0}, 2 b_{0}$, as follows from (6.13) and Theorem 2.4. Hence Theorem 6.3 is proved by (6.18) and the equations obtained by using (6.20) in (6.12) and (6.13).

Finally, to prove Theorem 6.4 , we use $(6.21),(6.13)$ in (6.12) and get

$$
y_{/ i j}=-\left(a_{1}+2 a_{2} y+3 a_{3} y^{2}\right) \bar{g}_{i j}, \quad z_{/ p q}=-\left(a_{1}-2 a_{2} z+2 a_{3} z^{2}\right) \bar{g}_{p q} .
$$

Since $\bar{a}=2\left(a_{2}+3 a_{3} y\right), \bar{b}=2\left(-a_{2}+3 a_{3} z\right)$ by (6.13), and $a_{3} \neq 0$, equations (6.7) and (6.22) can be expressed in terms of $\bar{a}, \bar{b}$. The result is readily found to be

$$
\tau=A(\bar{a}+\bar{b}),
$$




$$
\bar{a}_{/ i j}=-(1 / 2)\left(\bar{a}^{2}+B\right) \bar{g}_{i j}, \quad \bar{b}_{/ p q}=-(1 / 2)\left(\bar{b}^{2}+B\right) \bar{g}_{p q},
$$

where $A=1 /\left(6 a_{3}\right), B=4\left(a_{2}^{2}-3 a_{1} a_{3}\right)$. This completes the proof of Theorems 6.2-6.4.

\section{IMPROPERLY CONFORMALLY SEPARABLE TENSORS}

7.1. A preliminary theorem. The improperly conformally separable tensor with $\rho=1$ is

$$
*_{\alpha \beta}=\left[\begin{array}{cc}
g_{i j} & 0 \\
0 & \sigma^{-2} g_{p q}
\end{array}\right]
$$

$$
\begin{array}{r}
\alpha, \beta, \gamma, \epsilon=1, \cdots, m, \\
i, j, k, l=1, \cdots, n, \\
p, q, r, s=n+1, \cdots, m,
\end{array}
$$

where

$$
\sigma=\sigma\left(x^{\alpha}\right), \quad g_{i j}=g_{i j}\left(x^{k}\right), \quad g_{p q}=g_{p q}\left(x^{r}\right) .
$$

We suppose throughout this section that $\partial_{i} \sigma \neq 0$, that is, that ${ }^{*} g_{\alpha \beta}$ is not separable in the ordinary sense. For the tensor (7.1) we have (cf. (3.6))

$$
\begin{aligned}
& * R_{i p}=-(m-n-1) \partial_{p} \sigma_{i}, \\
& { }^{*} R_{i j}=R_{i j}+(m-n) \sigma\left(\frac{1}{\sigma}\right)_{, i j}, \\
& { }^{*} R_{p q}={ }^{\prime} R_{p q}+{ }^{*} g_{p q} g^{i j}\left[\sigma\left(\frac{1}{\sigma}\right)_{, i j}+(m-n-1) \sigma^{2}\left(\frac{1}{\sigma}\right)_{, i}\left(\frac{1}{\sigma}\right)_{, j}\right],
\end{aligned}
$$

where the comma denotes covariant differentiation with respect to $g_{i j}$. Let (7.1) represent an $E_{m}(c)$, so that

$$
{ }^{*} R_{\alpha \beta}=-(m-1) c^{*} g_{\alpha \beta},
$$

$c=$ const.

On account of this, $(7.2)_{1}$ becomes

$$
(m-n-1) \partial_{p} \sigma_{i}=0 .
$$

Hence

THEOREM 7.1. If the improperly conformally separable tensor (7.1) represents an $E_{m}$, then either $m=n+1$ or $\sigma$ is of the form $z\left(x^{r}\right) / y\left(x^{k}\right)$.

We discuss these two cases separately.

7.2. The case $m=n+1$. Since $\pm g_{m m}$ may be absorbed in $\sigma^{-2},(7.1)$ may be written

$$
*_{\alpha \beta}=\left[\begin{array}{cc}
g_{i j} & 0 \\
0 & e \sigma^{-2}
\end{array}\right], \quad e= \pm 1 .
$$

For this case ' $R_{m m}=0$, and, in consequence of (7.2), equation (7.3) is equivalent to 


$$
\begin{gathered}
R_{i j}+\sigma\left(\frac{1}{\sigma}\right)_{, i j}=-n c g_{i j}, \\
g^{i j} \sigma\left(\frac{1}{\sigma}\right)_{, i j}=-n c .
\end{gathered}
$$

If $n=2$, we have shown (Theorem 4.1) that $g_{i j}$ represents an $S_{2}$. We now consider the more general case when $n>1$ and the component tensor $g_{i j}$ is such that

$$
R_{i j}=-(n-1) a g_{i j}, \quad a=\text { const., }
$$

that is, that $g_{i j}$ represents an $E_{n}$ or an $S_{2}$. Because of (7.6), equation (7.5) becomes

$$
\left(\frac{1}{\sigma}\right)_{, i j}=-[n c-(n-1) a] \frac{1}{\sigma} g_{i j} .
$$

Transvecting this by $g^{i j}$ and comparing the result with $(7.5)_{2}$, we find that

$$
a=c .
$$

On account of this, equation (7.7) becomes

$$
\left(\frac{1}{\sigma}\right)_{, i j}=-\frac{a}{\sigma} g_{i j}
$$

Since (7.5) $)_{2}$ is evidently a consequence of (7.8) and (7.9), the latter equations, because of (7.6), are equivalent to (7.5), and hence to (7.3). Thus we have proved the following

THEOREM 7.2 (i). Let $g_{i j}$ be the fundamental tensor of an $E_{n}(a)$ or $S_{2}(a)$. Then in order that the improperly conformally separable tensor (7.4) of the type $(n>1, m-n=1)$ may represent an $E_{m}$, it is necessary and sufficient that $g_{i j}$ and $\sigma$ satisfy equation (7.9).

Since by supposition $g_{i j}$ represents an $E_{n}(a)$ or $S_{2}(a)$, it follows from (7.9) that the tensor $\sigma^{2} g_{i j}$ represents $E_{n}$ 's or $S_{2}$ 's (Theorem 2.1). If, in particular, $a=0$, then (7.9) reduces to $(1 / \sigma)_{, i j}=0$, and therefore the $E_{n}(0)$ or $S_{2}(0)$ with fundamental tensor $g_{i j}$ has a parallel vector field. Hence

THEOREM 7.2 (ii). If the improperly conformally separable tensor (7.4) and its first component tensor $g_{i j}$ represent an $E_{m}(c)$ and an $E_{n}(a)$ or $S_{2}(a)$, respectively, then the lensor $\sigma^{2} g_{i j}$ represents $E_{n}$ 's or $S_{2}$ 's and $c=a$. If $c=a=0$, then the $E_{n}(0)$ or $S_{2}(0)$ with fundamental tensor $g_{i j}$ possesses a parallel vector field.

Conversely, let $g_{i j}$ be the fundamental tensor of an $E_{n}(a)$ which is conformal $\left({ }^{17}\right)$ to another $E_{n}$, then the equation

(17) An $E_{n}$ with fundamental tensor $g_{i j}$ is said to be conformal to another $E_{n}$ if a nonconstant scalar $y$ exists such that $y^{-2} g_{i j}$ is the fundamental tensor of an $E_{n}$. 


$$
y_{, i j}=-(a y+f) g_{i j},
$$

$f=$ const.

has a solution for $y$ (cf. Theorems 2.1,2.3 and (2.11)). Thus, if $a \neq 0$, the function $1 / \sigma=y+f / a$ evidently satisfies (7.9). Hence we have the following converse to Theorem 7.2 (ii):

THEOREM 7.2 (iii). Given an $E_{n}$ whose scalar curvature is zero and which has a parallel vector field, or one whose scalar curvature is not zero and which is conformal to another $E_{n}$, then the given $E_{n}$ can be isometrically imbedded in an $E_{n+1}$ as a member of $\infty^{1}$ isometric, non-parallel, and totally geodesic hypersurfaces.

The present case has already been considered with a different method by Fialkow $\left[7,7^{\prime}\right]$, and the results stated in Theorems 7.2 (i), (ii) and (iii) are due to him. However $\left({ }^{18}\right)$, he overlooked the exceptional case $E_{n}(0)$, for which the property of its being conformal to another $E_{n}$ is not a sufficient condition for it to be imbeddable in an $E_{n+1}$ in the manner stated in Theorem 7.2 (iii).

7.3. The case $m-n>1$. By Theorem 7.1 , in this case $\sigma$ must be of the form $z\left(x^{r}\right) / y\left(x^{k}\right)$. Since $z\left(x^{r}\right)$ may be absorbed in $g_{p q}$, there is no loss of generality in assuming that $\sigma=1 / y\left(x^{k}\right)$. Thus the conformally separable tensor under consideration takes the form

$$
{ }^{*} g_{\alpha \beta}=\left[\begin{array}{cc}
g_{i j} & 0 \\
0 & y^{2} g_{p q}
\end{array}\right],
$$

where $y \neq$ const. On account of (7.3) and the fact that the fundamental tensors $g_{p q}$ and ${ }^{*} g_{p q}=y^{2} g_{p q}$ have identical Ricci tensors, equation (7.2) $)_{3}$ for the tensor (7.10) can be written

(7.11) $R_{p q}={ }^{\prime} R_{p q}=-\left\{(m-1) c+g^{i j}\left[\frac{y_{, i j}}{y}+(m-n-1) \frac{y_{, i} y_{. i}}{y^{2}}\right]\right\} y^{2} g_{p q}$.

(18) Equations (3.11) of Fialkow [7] represent a necessary and sufficient condition for an $E_{n}$ to be imbeddable in an $E_{n+1}$ as a member of $\infty^{1}$ isometric, non-parallel, totally geodesic hypersurfaces. In p. 427 (line 18) of the same paper, we find the sentence "According to Brinkmann (3.11) is the necessary and sufficient condition that $E_{n}$ be conformal to another Einstein space

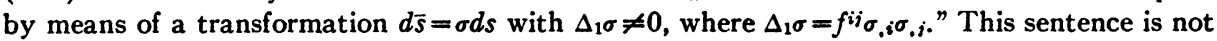
entirely correct; in fact, the condition is sufficient but not necessary. To explain, we use Fialkow's notation. Brinkmann's original necessary and sufficient condition referred to above is $[3$, p. 125 , Theorem II $]$ that a coordinate system exists in which the fundamental tensor of $E_{n}$ is of the form

$$
\begin{aligned}
f_{n n} & =\left(c x^{n^{2}}+2 A x^{n}+d\right)^{-1}, \\
f_{\lambda \mu} & =\left(c x^{n^{2}}+2 A x^{n}+d\right) F_{\lambda \mu}\left(x^{y}\right), \quad f_{n \lambda}=0, \\
& A \text { and } d \text { constant, }
\end{aligned}
$$

and the form $F_{\lambda \mu}\left(x^{\nu}\right) d x^{\lambda} d x^{\mu}$ is the fundamental form of an $E_{n-1}$. Equations (3.11) of Fialkow [7] differ from $(\Delta)$ by the absence of the constant $A$, and, $(\Delta)$ can be reduced, by putting $x^{n}=x^{n}+B, B=$ const., to (3.11) of Fialkow [7] when $c \neq 0$ but not when $c=0$. This justifies our statement. 
Hence

THEOREM 7.3. If the improperly conformally separable tensor (7.10) of the type $(n, m-n>2)$ represents an $E_{m}$, then its second component tensor $*_{p q}=y^{2} g_{p q}$ represents $E_{m-n}$ 's.

We shall now consider separately the following three subcases: (1) $n=1$; (2) $n>1$ and $g_{i j}$ represents an $E_{n}$ or $S_{2} ;$ (3) $n=2$.

Subcase 1. $n=1, m-n>1$.

The conformally separable tensor in question is

$$
{ }^{*} g_{\alpha \beta}=\left[\begin{array}{cc}
g_{11} & 0 \\
0 & \cdot y^{2} g_{p q}
\end{array}\right], \quad y=y\left(x^{1}\right) \neq \text { const. }
$$

With $n, x, g_{11}, y^{2} g_{p q}$ in place of $m-n, x^{m}, e \sigma^{-2}, \rho^{-2} g_{i j}$, respectively, the case considered in $\$ 4$ reduces to the present subcase. Hence from Theorems 4.1 and 4.2 , we have

THEOREM 7.4. In order that the improperly conformally separable tensor (7.12) with $m>2$ may represent an $E_{m}(c)$, it is necessary and sufficient that $g_{p_{q}}$ represent an $E_{m-1}(b)$ or $S_{2}(b)$ and

$$
g_{11}=\frac{\left(y^{\prime}\right)^{2}}{b-c y^{2}} .
$$

This result can also be proved directly from (7.2) and (7.3).

Subcase 2. $n>1, m-n>1$, and $g_{i j}$ represents an $E_{n}$ or $S_{2}$.

By hypothesis, we have, besides (7.2), (7.3), (7.11), also

$$
R_{i j}=-(n-1) a g_{i j},
$$

$$
a=\text { const. }
$$

In consequence of this and (7.3), equation (7.2) 2 becomes

$$
y_{, i j}=-\frac{(m-1) c-(n-1) a}{m-n} y g_{i j} .
$$

This equation is of the form (2.1). And since (7.14) is satisfied, it follows from $(2.11)_{1}$ that (cf. Theorem 2.3)

$$
\frac{(m-1) c-(n-1) a}{m-n} y=a y+f, \quad f=\text { const., }
$$

which give $f=0$ and $a=c$. Therefore (7.15) is equivalent to

$$
\begin{aligned}
y_{, i j} & =-a y g_{i j}, \\
c & =a .
\end{aligned}
$$

As a consequence of (7.14) and (7.16), $\bar{g}_{i j}=y^{-2} g_{i j}$ is the fundamental tensor of an $E_{n}(\bar{a})$ or $S_{2}(\bar{a})$, where $\bar{a}$ is determined from (cf. Theorem 2.4) 


$$
g^{i j} y_{, i} y_{, j}=-\left(a y^{2}+\bar{a}\right) .
$$

When (7.16)-(7.18) are used in (7.11), the latter becomes

$$
R_{p q}=-(m-n-1)(-\bar{a}) g_{p q},
$$

which shows that $g_{p q}$ represents an $E_{m-n}(-\bar{a})$ or $S_{2}(-\bar{a})$. Hence

THEOREM 7.5 (i). If the improperly conformally separable tensor (7.10) of the type $(n>1, m-n>1)$ and its component tensor $g_{i j}$ represent, respectively, an $E_{m}(c)$ and an $E_{n}(a)$ or $S_{2}(a)$, then (1) $g_{p q}$ represents an $E_{m-n}(b)$ or $S_{2}(b)$, (2) $y^{-2} g_{i j}$ represents an $E_{n}(\bar{a})$ or $S_{2}(\bar{a})$, and (3) $c=a, b=-\bar{a}$. If $c=a=0$, the $E_{n}(0)$ or $S_{2}(0)$ with fundamental tensor $g_{i j}$ possesses a parallel vector field.

We observe that when (7.14) is supposed, equations (7.2), (7.3) are equivalent to (7.16)-(7.19). Then, by a consideration similar to that leading to Theorem 7.2 (iii), we can prove the following

THEOREM 7.5 (ii). Given an $E_{n}$ whose scalar curvature is zero and which possesses a parallel vector field or one whose scalar curvature is not zero and which is conformal to another $E_{n}$, then the given $E_{n}$ can be isometrically imbedded in an $E_{m}$ of any dimension $m$ greater than $n+1$ as a member of $\infty^{m-n}$ isometric and totally geodesic subspaces $E_{n}$ 's which are orthogonal to $\infty^{n}$ totally umbilical subspaces $E_{m-n}$ 's or $S_{2}$ 's.

Subcase 3. $n=2, m-n>1$.

For this case we have

$$
R_{i j}=-a\left(x^{k}\right) g_{i j}
$$

In consequence of this and (7.3), equation $(7.2)_{2}$ becomes

$$
y_{, i j}=-\frac{(m-1) c-a\left(x^{k}\right)}{m-2} y g_{i j}
$$

This is of the form (2.1), and consequently, it follows from (2.9) that (cf. Theorem 2.3)

$$
a\left(x^{k}\right)=a(y)=\frac{d}{d y}\left[\frac{(m-1) c-a(y)}{m-2} y\right]
$$

that is,

$$
a^{\prime}(y) y+(m-1) a(y)=(m-1) c .
$$

Multiplying this by $y^{m-2}$ and then integrating, we have

$$
a(y)=c-(m-2) f y^{1-m}, \quad f=\text { const. }
$$

Here it is evident that $a(y)$ is constant or not according as $f$ is or is not zero.

With this value of $a(y)$, equation (7.21) becomes 


$$
y_{, i j}=-\left(c y+f y^{2-m}\right) g_{i j} \text {. }
$$

From this and (2.10), which holds by Theorem 2.3, it follows that

$$
g^{i j} y_{, i} y_{, j}=-\left(c y^{2}+\frac{2 f}{3-m} y^{3-m}-b\right), \quad b=\text { const. }
$$

When (7.23) and (7.24) are substituted in (7.11) for $n=2$, the result is

$$
R_{p q}=-(m-3) b g_{p q},
$$

which is the condition for $g_{p q}$ to represent an $E_{m-2}(b)$ or $S_{2}(b)$. Hence

THEOREM 7.6. In order that the improperly conformally separable tensor (7.10) of the type $(n=2, m-n>1)$ may represent an $E_{m}(c)$, it is necessary and sufficient that (1) $g_{i j}$ and $y$ be such that equation (7.23) is satisfied with a constant $f$, and (2) $g_{p q}$ represent an $E_{m-2}$ or $S_{2}$ of scalar curvature b given by (7.24).

As a verification we observe that the result for subcase 2 with $n=2$ is identical with the result for subcase 3 with $a=$ const. (that is, $f=0$ ).

Finally, it follows from the last but one paragraph of $\$ 2$ that a 2-dimensional fundamental tensor $g_{i j}$ actually exists whose scalar curvature is not constant and for which equation (7.23) admits a solution for $y$. Thus the existence of an improperly conformally separable tensor which represents an $E_{m}$ and whose component tensors $g_{i j}$ and ${ }^{*} g_{p q}$ are such that, the first represents a $V_{2}$ which is not an $S_{2}$, while the second represents $E_{m-2}$ 's or $S_{2}$ 's. This fact is in contrast with Theorem 6.2 of the preceding section and Theorem 8.1 of the following section.

8. Separable tensors. In this section we reproduce some results of Fialkow concerning a separable tensor, thus completing our discussion of the conformally separable tensor which represents an $E_{m}$ and each of whose component tensors either is of dimension less than 3 or represents $E$ 's.

For the separable tensor

$$
*_{\alpha \beta}=\left[\begin{array}{cc}
g_{i j} & 0 \\
0 & g_{p q}
\end{array}\right]
$$

equations $(\dot{3} .6)$ reduce to

$$
* R_{i p}=0, \quad * R_{i j}=R_{i j}, \quad * R_{p q}=R_{p q} .
$$

Suppose that (8.1) represents an $E_{m}(c)$, that is, that

$$
{ }^{*} R_{\alpha \beta}=-(m-1) c{ }^{*} g_{\alpha \beta},
$$$$
c=\text { const. }
$$

Then according as $n>1, m-n>1$, or $n>1, m-n=1$, (8.2) become

$$
0=0, \quad R_{i j}=-(m-1) c g_{i j}, \quad R_{p q}=-(m-1) c g_{p q} ;
$$

or 


$$
0=0, \quad R_{i j}=-(m-1) c g_{i j}, \quad 0=-(m-1) c g_{m m} .
$$

From these we have at once the following results due to Fialkow [7]:

THEOREM 8.1. A separable tensor of the type $(n>1, m-n>1)$ represents an $E_{m}(c)$, if and only if its component tensors $g_{i j}$ and $g_{p q}$ represent, respectively, an $E_{n}(a)$ or $S_{2}(a)$ and an $E_{m-n}(b)$ or $S_{2}(b)$, and $(m-1) c=(n-1) a=(m-n-1) b$.

TheOREM 8.2. A separable tensor of the type $(n>1, m-n=1)$ represents an $E_{m}(c)$, if and only if its component tensor $g_{i j}$ represents an $E_{n}(0)$ or $S_{2}(0)$. Then $c=0$.

\section{Particular Cases}

9. $S_{m}$ with conformally separable fundamental tensor. It is well known that the fundamental form of an $S_{m}(c)$ can always be reduced to the Riemann form $[5$, p. 85$]$

$$
\frac{e_{1}\left(d x^{1}\right)^{2}+\cdots+e_{m}\left(d x^{m}\right)^{2}}{\left\{1+(c / 4)\left[e_{1}\left(x^{1}\right)^{2}+\cdots+e_{m}\left(x^{m}\right)^{2}\right]\right\}^{2}}
$$

where each $e$ is \pm 1 . The form (9.1) is evidently properly conformally separable $\left({ }^{19}\right)$ of the type $(n, m-n)$, where $n$ may be any integer from 1 to $m-1$. In what follows we give a few theorems concerning a conformally separable tensor which represents an $S_{m}$. Throughout this section all the symbols and indices have the same usual meaning.

From (3.4) it follows that the condition

$$
{ }^{*} R_{\alpha \beta \gamma}^{\epsilon}=c\left(\delta_{\beta}^{\epsilon}{ }^{*} g_{\alpha \gamma}-\delta_{\gamma}^{\epsilon} * g_{\alpha \beta}\right)
$$

for the conformally separable tensor (1.1) to represent an $S_{m}$ becomes

$$
\begin{aligned}
& { }^{\prime} R_{i j k}^{l}=\left(c+\rho^{p} \rho_{p}\right)\left(\delta_{j}^{l} * g_{i k}-\delta_{k}^{l} * g_{i j}\right), \\
& { }^{\prime} R_{p q r}^{s}=\left(c+\sigma^{i} \sigma_{i}\right)\left(\delta_{q}^{8} *_{g_{p r}}-\delta_{r}^{8} *_{p q}\right), \\
& (n-1)\left(\partial_{i} \rho_{p}+\rho_{p} \sigma_{i}\right)=0, \quad(m-n-1)\left(\partial_{p} \sigma_{i}+\rho_{p} \sigma_{i}\right)=0 \text {, } \\
& \rho\left(\frac{1}{\rho}\right)_{; p q}=-\left[c+\frac{1}{n} *^{i j \sigma}\left(\frac{1}{\sigma}\right)_{; i j}\right] * g_{p q} \text {, } \\
& \sigma\left(\frac{1}{\sigma}\right)_{; i j}=-\left[c+\frac{1}{m-n} *^{*} g^{p q} \rho\left(\frac{1}{\rho}\right)_{; p q}\right] * g_{i j} .
\end{aligned}
$$

For $\rho=\sigma=1$, we have immediately $[9$, p. 896]:

THEOREM 9.1. A separable tensor cannot represent an $S_{m}$ of nonzero scalar curvature.

(19) The fundamental form ${ }^{*} g_{\alpha \beta} d x^{\alpha} d x^{\beta}$ is said to be conformally separable, if the tensor $g_{\alpha \beta}$ is conformally separable, and vice versa. 
From $(9.3)_{1}$ and $(9.3)_{2}$ we have the following theorem [14]:

THEOREM 9.2. If a conformally separable tensor represents an $S_{m}$, then each of its component tensors, if it is of dimension greater than 2, represents $S$ 's.

Since an $S$ is necessarily an $E$, it follows from this theorem that all properties of the conformally separable tensor which represents an $E_{m}$ and each of whose component tensors either is of dimension less than 3 or represents $E$ 's must also be shared by the conformally separable tensor which represents an $S_{m}$. This observation together with what we have obtained in $\$ \S 4-7$ enables us to prove the following extension of Theorem 9.2:

THEOREм 9.3. If a conformally separable tensor ${ }^{*} g_{\alpha \beta}$ which is not an ordinarily separable tensor represents an $S_{m}$, then each of its component tensors either is of dimension 1 or represents $S$ 's. Conversely, if ${ }^{*} g_{\alpha \beta}$ represents an $E_{m}$ and each of its component tensors either is of dimension 1 or represents $S$ 's, then the $E_{m}$ is an $S_{m}$.

Proof. The proof of this theorem has to be carried out separately for several cases. Consider first the case of the properly conformally separable tensor, which we subdivide into the following three types: (1) $n=2, m-n=1$, (2) $n>2, m-n=1$, (3) $n>1, m-n>1$.

For the type $(n=2, m-n=1)$, Theorem 9.3 reduces to Theorem 4.1 for $m=2$, because an $E_{3}$ is an $S_{3}$.

For the type $(n>2, m-n=1)$, equations (9.3) become

$$
\begin{aligned}
{ }^{\prime} R_{i j k}^{l}= & \left(c+\rho^{m} \rho_{m}\right)\left(\delta_{j}^{l} * g_{i k}-\delta_{k}^{l} * g_{i j}\right), \\
& \partial_{i} \rho_{m}+\rho_{m} \sigma_{i}=0 \\
\rho\left(\frac{1}{\rho}\right)_{; m m}=- & {\left[c+\frac{1}{n}{ }^{*} g^{i j} \sigma\left(\frac{1}{\sigma}\right)_{i i j}\right] * g_{m m}, } \\
\sigma\left(\frac{1}{\sigma}\right)_{; i j}=- & {\left[c+{ }^{*} g^{m m} \rho\left(\frac{1}{\rho}\right)_{; m m}\right] * g_{i j} . }
\end{aligned}
$$

The first part of the theorem follows at once from (9.4) $)_{1}$. To prove the converse part, we suppose that ${ }^{*} g_{\alpha \beta}$ and its component tensor ${ }^{*} g_{i j}=\rho^{-2} g_{i j}$ represent, respectively, an $E_{m}$ and $S_{n}$ 's, and show that (9.4) are satisfied. From this supposition it follows that all the equations appearing in $\$ 4$ (with the exception of $\left.\left(4.6^{\prime}\right)\right)$ and the equation

$$
{ }^{\prime} R_{i j k}^{l}=*_{a}\left(x^{m}\right)\left(\delta_{j}^{l} *_{i k}-\delta_{k}^{l} *_{i j}\right)
$$

are true. Now equations $(9.4)_{2}$ and $(9.4)_{3}$ are satisfied because of $(4.2)_{1},(4.2)_{2}$ and (4.3). And on account of (9.5), equation (9.4) 1 becomes 


$$
c=* a\left(x^{m}\right)-*^{m m}\left(\rho_{m}\right)^{2},
$$

which, by Theorem 4.1, is identical with (4.6). Finally, by virtue of (4.15), equation $(9.4)$, becomes $\left(4.14^{\prime}\right)$. Thus equations (9.4) are satisfied, as was to be proved.

We can now come to the properly conformally separable tensor of the type $(n>1, m-n>1)$. As a consequence of Theorems 9.2 and 5.2, a properly conformally separable tensor of this type which represents an $S_{m}$ must be of the form

$$
*_{g_{\alpha \beta}}=\tau^{-2}\left[\begin{array}{cc}
\bar{g}_{i j} & 0 \\
0 & \bar{g}_{p q}
\end{array}\right] \text {. }
$$

The Riemann tensors ${ }^{*} R_{\alpha \beta \gamma}^{e}$; and " $R_{\alpha \beta \gamma}^{e}$ of the tensors ${ }^{*} g_{\alpha \beta}$ and

$$
{ }^{\prime \prime} g_{\alpha \beta}=\left[\begin{array}{cc}
\bar{g}_{i j} & 0 \\
0 & \bar{g}_{p q}
\end{array}\right]
$$

are connected by (cf. (2.5))

$$
\begin{aligned}
* R_{\alpha \beta \gamma}^{\epsilon}= & " R_{\alpha \beta \gamma}^{\epsilon}+\left(\delta_{\beta}^{\epsilon} \tau_{: \alpha \gamma}-\delta_{\gamma}^{\epsilon} \tau_{: \alpha \beta}\right) \tau^{-1}+"{ }^{k \epsilon}{ }^{\kappa \epsilon}\left(\tau_{: \alpha \beta}{ }^{\prime \prime} g_{\alpha \gamma}-\tau_{: \alpha \gamma} " g_{\alpha \beta}\right) \tau^{-1} \\
& -" g^{\alpha \lambda} \tau_{: \kappa} \tau_{: \lambda}\left(\delta_{\beta}^{\epsilon} " g_{\alpha \gamma}-\delta_{\gamma}^{\epsilon} " g_{\alpha \beta}\right) \tau^{-2},
\end{aligned}
$$

where the colon denotes covariant differentiation with respect to " $g_{\alpha \beta}$; the indices $\alpha, \beta, \gamma, \epsilon, \kappa, \lambda$ have the range $1, \cdots, m$; and the components of " $R_{\alpha \beta \gamma}^{e}$ are (cf. (9.7), (3.4))

$$
\begin{gathered}
\text { " } R_{i j k}^{l}=\bar{R}_{i j k}^{l}, \quad "{ }^{\prime} R_{p q r}^{*}=\bar{R}_{p q r}^{*}, \\
" R_{\alpha \beta \gamma}^{e}=0 \quad \text { if } \alpha, \beta, \gamma, \epsilon \text { are not all in the same range. }
\end{gathered}
$$

From Theorem 9.2 and the remark below it and also the equations in $\S 6$, it follows that if the tensor ${ }^{*} g_{\alpha \beta}$ defined by $(9.6)$ represents an $S_{m}(c)$, then we have

$$
\begin{array}{rlrl}
\tau=y\left(x^{k}\right)+z\left(x^{r}\right), & \\
M & =a_{0}+a_{1} y+a_{2} y^{2}+a_{3} y^{3}, & T & =b_{0}+a_{1} z-a_{2} z^{2}+a_{3} z^{3}, \\
y_{/ i j} & =-M^{\prime} \bar{g}_{i j}, & z_{/ p q} & =-T^{\prime} \bar{g}_{p q}, \\
\bar{R}_{i j k}^{l} & =M^{\prime \prime}\left(\delta_{j}^{l} \bar{g}_{i k}-\delta_{k}^{l} \bar{g}_{i j}\right), & \bar{R}_{p q r}^{8} & =T^{\prime \prime}\left(\delta_{q}^{8} \bar{g}_{p r}-\delta_{r}^{8} \bar{g}_{p q}\right),
\end{array}
$$

where $a_{3}=0$ unless $m=2 n=4$. It can now be readily verified that in consequence of (9.8)-(9.10), equation (9.2) becomes $a_{3}=0$. On account of this, it follows easily from (9.10) that if any of the component tensors $\tau^{-2} \bar{g}_{i j}$ and $\tau^{-2} \bar{g}_{p q}$ is of dimension 2, then it represents $S_{2}$ 's. The first part of Theorem 9.3 for the present case is thus proved. We now suppose that ${ }^{*} g_{\alpha \beta}$ represents an $E$ and its component tensors $S$ 's. Then (9.10) are satisfied with $a_{3}=0$, and 
consequently, equation (9.2) is satisfied, as follows from the sentence below (9.10). Hence the $E_{m}$ in question is an $S_{m}$, and the proof of our theorem for the case of a properly conformally separable tensor is completed.

Finally, for an improperly conformally separable tensor with $\rho=1, \partial_{i} \sigma \neq 0$, equations (9.3) become

$$
\begin{gathered}
R_{i j k}^{l}=c\left(\delta_{j g_{i k}}^{l}-\delta_{k}^{l} g_{i j}\right), \\
{ }^{\prime} R_{p q r}^{s}=\left(c+\sigma^{i} \sigma_{i}\right)\left(\delta_{q}^{8}{ }^{*} g_{p r}-\delta_{r}^{8}{ }^{*} g_{p q}\right), \\
(m-n-1) \partial_{p} \sigma_{i}=0 \\
\sigma\left(\frac{1}{\sigma}\right)_{, i j}=-c g_{i j} .
\end{gathered}
$$

If we compare these equations with those appearing in $\$ 7$, we shall see easily that Theorem 9.3 is true. Theorem 9.3 has thus been completely proved.

10. $E_{4}$ with conformally separable fundamental tensor of the type $(2,2)$. Let us review the cases we have considered for a conformally separable tensor which represents an $E_{4}$.

The type $(3,1)$. We have considered only the particular case when the first component tensor $g_{i j}$ represents $E_{3}$ 's, that is, $S_{3}$ 's. By Theorem 9.3, an $E_{4}$ with such a conformally separable fundamental tensor is an $S_{4}$.

The type $(2,2)$. If $*_{\alpha \beta}$ is a properly conformally separable tensor, then either its component tensors both represent $S_{2}$ 's or neither of them does (cf. Theorem 6.2); for the respective cases, the $E_{4}$ is an $S_{4}$ or not an $S_{4}$ (cf. Theorem 9.3). If ${ }^{*} g_{\alpha \beta}$ is an improperly conformally separable tensor with $\rho=1, \partial_{i} \sigma \neq 0$, the component tensor ${ }^{*} g_{p q}$ necessarily represents $S_{2}$ 's, while the other, $g_{i j}$, may or may not (cf. Theorem 7.6); for the respective cases, the $E_{4}$ is an $S_{4}$ or not an $S_{4}$. Finally, if ${ }^{*} g_{\alpha \beta}$ is a separable tensor, its two component tensors each represent an $S_{2}$ of equal nonzero scalar curvature (cf. Theorems 8.1 and 9.1).

Hence we have three and only three cases in which the $E_{4}$ is not an $S_{4}$; they are the cases of Theorem 6.4, Theorem 7.6 for $m=4$ and $f \neq 0$, and Theorem 8.1 for $m=2 n=4$ and $a \neq 0$. From these theorems and (2.14), (9.1) we have at once the following

THEOREM 10.1. A conformally separable fundamental form of the type $(2,2)$ represents an $E_{4}$ which is not an $S_{4}$, if and only if it can be reduced to one of the following forms (in which each $e$ is $\pm 1 ; A, B, C, D$ are constants, and $A \neq 0$ ):

$$
\begin{array}{r}
A\left(x^{1}+x^{3}\right)^{-2}\left\{\frac{\left(d x^{1}\right)^{2}}{(1 / 3)\left(x^{1}\right)^{3}+B x^{1}+C}+e_{2}\left[(1 / 3)\left(x^{1}\right)^{3}+B x^{1}+C\right]\left(d x^{2}\right)^{2}\right. \\
\left.+\frac{\left(d x^{3}\right)^{2}}{(1 / 3)\left(x^{3}\right)^{3}+B x^{3}+D}+e_{4}\left[(1 / 3)\left(x^{3}\right)^{3}+B x^{3}+D\right]\left(d x^{4}\right)^{2}\right\},
\end{array}
$$




$$
\frac{\left(d x^{1}\right)^{2}}{C\left(x^{1}\right)^{2}+A\left(x^{1}\right)^{-1}+B}+e_{2}\left[C\left(x^{1}\right)^{2}+A\left(x^{1}\right)^{-1}+B\right]\left(d x^{2}\right)^{2}
$$

$$
+\frac{\left(x^{1}\right)^{2}\left[e_{3}\left(d x^{3}\right)^{2}+e_{4}\left(d x^{4}\right)^{2}\right]}{\left[1+(B / 4)\left(e_{3}\left(x^{3}\right)^{2}+e_{4}\left(x^{4}\right)^{2}\right)\right]^{2}},
$$

(3) $\frac{e_{1}\left(d x^{1}\right)^{2}+e_{2}\left(d x^{2}\right)^{2}}{\left[1+(A / 4)\left(e_{1}\left(x^{1}\right)^{2}+e_{2}\left(x^{2}\right)^{2}\right)\right]^{2}}+\frac{e_{3}\left(d x^{3}\right)^{2}+e_{4}\left(d x^{4}\right)^{2}}{\left[1+(A / 4)\left(e_{3}\left(x^{3}\right)^{2}+e_{4}\left(x^{4}\right)^{2}\right)\right]^{2}}$.

Form (3) has been obtained by Kasner [11], and a form which is essentially the same as (2) for $B=1$ by Kottler [12, p. 443]. Form (1) however seems to be introduced here for the first time.

\section{REFERENCES}

1. E. Bompiani, Spazi Riemanniani luoghi di totalmente geodetiche, Rend. Circ. Mat. Palermo vol. 48 (1924) pp. 121-134.

2. H. W. Brinckmann, Riemann spaces conformal to Einstein spaces, Math. Ann. vol. 91 (1924) pp. 269-278.

3. - Einstein spaces which are mapped conformally on each other, ibid. vol. 94 (1925) pp. 119-145.

4. A. Delgleize, Sur les equations de Weingarten et les espaces pseudosphériques, Bulletin de la Societé royale des Sciences de Liège vol. 4 (1935) pp. 158-161.

5. L. P. Eisenhart, Riemannian geometry, 1926, Princeton.

6. A. Fialkow, Einstein spaces in a space of constant curvature, Proc. Nat. Acad. Sci. U.S.A. vol. 24 (1938) pp. 30-34. 428.

7. — , Totally geodesic Einstein spaces, Bull. Amer. Math. Soc. vol. 45 (1939) pp. 423-

7'. - Correction to "Totally geodesic Einstein spaces," ibid. vol. 48 (1942) pp. 167-168.

8. - Conformal geodesics, Trans. Amer. Math. Soc. vol. 45 (1939) pp. 443-473.

9. F. A. Ficken, The Riemannian and affine differential geometry of product-spaces, Ann. of Math. (2) vol. 40 (1939) pp. 892-913.

10. E. Kasner, An algebraic solution of the Einstein equations, Trans. Amer. Math. Soc. vol. 27 (1925) pp. 101-105.

11. - Separable quadratic differential forms and Einstein solutions, Proc. Nat. Acad. Sci. U.S.A. vol. 11 (1925) pp. 95-96.

12. F. Kottler, Über die physikalischen Grundlagen der Einsteinsch Gravitationstheorie, Annalen der Physik (4) vol. 56 (1918) pp. 401-462.

13. J. A. Schouten, and D. J. Struik, On some properties of general manifolds relating to Einstein's theory of gravitation, Amer. J. Math. vol. 43 (1921) pp. 217-221.

14. K. Yano, Conformally separable quadratic differential forms, Proc. Imp. Acad. Tokyo vol. 16 (1940) pp. 83-86.

- 15. - Concircular geometry, I. Concircular transformations, loc. cit. pp. 195-200. 360.

16. - Concircular geometry, II. Integrability conditions of $\rho_{\mu \nu}=\phi g_{\mu \nu}$, loc. cit. pp. 354-

17. Yung-Chow Wong, Family of totally umbilical hypersurfaces in an Einstein space, Ann. of Math. (to appear).

Cambridge, Mass. 